JOURNAL OF

SYMPLECTIC GEOMETRY

Volume 3, Number 2, 171-219, 2005

\title{
LAGRANGIAN SUBMANIFOLDS AND LEFSCHETZ PENCILS
}

\author{
Denis Auroux, Vicente Muñoz, and Francisco Presas \\ Given a Lagrangian submanifold in a symplectic manifold and a \\ Morse function on the submanifold, we show that there is an iso- \\ topic Morse function and a symplectic Lefschetz pencil on the manifold \\ extending the Morse function to the whole manifold. From this con- \\ struction, we define a sequence of symplectic invariants classifying the \\ isotopy classes of Lagrangian spheres in a symplectic 4-manifold.
}

\section{Introduction}

For a symplectic manifold $(M, \omega)$, S. Donaldson has proved in $[\mathbf{1 0}]$ the existence of symplectic Lefschetz pencils using the recently introduced asymptotically holomorphic techniques $[\mathbf{2}, \mathbf{9}]$.

To give the definition of a Lefschetz pencil, recall that a chart $(\psi, U)$, $\psi=\left(z_{1}, \ldots, z_{n}\right): U \subset M \rightarrow \mathbb{C}^{n}$ is adapted at a point $p \in M$ if $\psi(p)=0$ and $\psi^{*}\left(J_{0}\right)$ is tamed by $\omega$ (where $J_{0}$ is the standard complex structure on

$\left.\mathbb{C}^{n}\right)$; equivalently, this means that complex lines in the local coordinates are symplectic with respect to $\omega$.

Definition 1.1. A symplectic Lefschetz pencil associated to a symplectic manifold $(M, \omega)$ consists of the following data:

(i) A codimension 4 symplectic submanifold $N$.

(ii) A surjective map $\phi: M-N \rightarrow \mathbb{C P}^{1}$.

(iii) A finite set of points $\Delta \subset M-N$ away from which the map $\phi$ is a submersion.

Moreover the data satisfy the following local models

(i) For any point $p \in N$, there exists an adapted chart $\left(z_{1}, \ldots, z_{n}\right)$ for which the submanifold $N$ has local equation $\left\{z_{1}=z_{2}=0\right\}$ and such that $\phi\left(z_{1}, \ldots, z_{n}\right)=z_{2} / z_{1}$. 
(ii) For any point $p \in \Delta$, there exists an adapted chart $\left(z_{1}, \ldots, z_{n}\right)$ in which we can write $\phi\left(z_{1}, \ldots, z_{n}\right)=z_{1}^{2}+\cdots+z_{n}^{2}+c$.

The main result of $[\mathbf{1 0}]$ is

Theorem 1.2 (Theorem 2 in $[\mathbf{1 0}]$ ). Let $(M, \omega)$ be a symplectic manifold such that the cohomology class $[\omega] / 2 \pi$ admits an integer lift $h$ in $H^{2}(M, \mathbb{Z})$. Then there exists a symplectic Lefschetz pencil whose fibers are homologous to the Poincaré dual of $k h$, for $k$ large enough.

The Lefschetz pencils obtained by Theorem 1.2 will be called Donaldson's Lefschetz pencils. For $k$ fixed large enough, they lie in a distinguished isotopy class of Lefschetz pencils. Moreover, they enjoy various remarkable topological and geometric properties (see, e.g., [1]). Our main result relates the geometry of these pencils to that of Lagrangian submanifolds. More precisely, the result that we want to prove is

Theorem 1.3. Let $(M, \omega)$ be an integral symplectic manifold. Let $\mathcal{L}$ be a Lagrangian submanifold of $M$ and let $f: \mathcal{L} \rightarrow[0,1]$ be a Morse function. Then there exists a sequence of Donaldson's Lefschetz pencils $\phi_{k}: M-$ $B_{k} \rightarrow \mathbb{C P}^{1}$ with $B_{k}$ and $\mathcal{L}$ disjoint, such that, for $k$ large, $\phi_{k}(\mathcal{L})$ is a smooth embedded arc in $\mathbb{C P}^{1}$. Moreover, there exists a parametrization of this arc $\gamma_{k}:[0,1] \rightarrow \phi_{k}(\mathcal{L})$ in such a way that $\left.\left(\gamma_{k}^{-1} \circ \phi_{k}\right)\right|_{\mathcal{L}}$ is a Morse function isotopic to $f$.

Recall that we mean that two Morse functions are isotopic if they are isotopic among Morse functions, i.e., there exists a 1-parametric family of (non-degenerate) Morse functions connecting them.

Statements similar to Theorem 1.3 have been part of the mathematical folklore for the past few years, after Donaldson suggested such a picture; however, to our knowledge the details of the statement had not been worked out, and our proof shows that some rather unexpected technical complications do occur. It is also worth mentioning the relationship between our result and the work of Seidel, who showed that if two vanishing cycles of a Lefschetz pencil can be joined by a "matching path" (see Section 8), then the total space of the pencil contains a Lagrangian sphere fibered over an arc in $\mathbb{C P}^{1}$; in the case where $\mathcal{L} \cong S^{n}$ and $f$ is a Morse function with only two critical points, our result can therefore be thought of as a converse to Seidel's construction.

It is possible to adapt our result to $S^{1}$-valued Morse functions. The proof will follow exactly the same pattern and we do not give the details but leave them to the careful reader.

The task of the next part of the article is to construct an equivalence of sets between Hamiltonian isotopy classes of Lagrangian spheres and the set of matching paths modulo a natural action of the fundamental group of the 
space of Lefschetz pencils. The precise result will be stated in Section 9 . For this we will use a parametric version of Theorem 1.3.

Theorem 1.4. Let $(M, \omega)$ be an integral symplectic manifold. Let $\left\{\mathcal{L}_{t}\right\}$ be a 1-parametric family of simply connected Lagrangian submanifolds of $M$, and let $\phi_{k, 0}$ and $\phi_{k, 1}$ be two sequences of Donaldson pencils obtained using the construction of Theorem 1.3, adapted to the submanifolds $\mathcal{L}_{0}$ and $\mathcal{L}_{1}$ and two Morse functions $f_{j}: \mathcal{L}_{j} \rightarrow[0,1](j=0,1)$. Assume moreover that $f_{0}$ and $f_{1}$ are isotopic through a family of Morse functions $f_{t}$. Then there exists a sequence of families of Donaldson pencils $\phi_{k, t}$ adapted to $\mathcal{L}_{t}$ and $f_{t}$ and coinciding with $\phi_{k, 0}$ and $\phi_{k, 1}$ at the ends.

The proof will be an extension of the non-parametric case; both cases rely on a local Lemma, extending previous results of Donaldson, that will be proved in Section 7 .

Finally, in Section 10, we define the group of automorphisms $\Gamma(\phi)$ of a Lefschetz pencil and discuss its properties. In particular, we show the asymptotic surjectivity as $k \rightarrow \infty$ of a natural homomorphism $\rho: \Gamma(\phi) \rightarrow$ $\pi_{0} \operatorname{Symp}(M, \omega)$, and exhibit various natural elements of its kernel. We also discuss the implications for matching paths and the relation with the projective dual of the discriminant curve of a projection to $\mathbb{C P}^{2}$.

\section{Asymptotically holomorphic tools}

Let $(M, \omega)$ be a symplectic manifold such that $[\omega / 2 \pi] \in H^{2}(M ; \mathbb{R})$ admits a lift to an integer cohomology class and let $h \in H^{2}(M ; \mathbb{Z})$ be one such lifting. In this case there is an hermitian line bundle $L$ with first Chern class $c_{1}(L)=h$, and we can equip $L$ with a hermitian connection $\nabla$ of curvature $-i \omega$.

Let $J$ be an almost complex structure on $M$ compatible with $\omega$ and let $g(u, v)=\omega(u, J v)$ be the associated metric. We define the sequence of rescaled metrics $g_{k}=k g$ with associated distance functions $d_{k}$. We give the following.

Definition 2.1. Let $E_{k}$ be a sequence of hermitian vector bundles with connection on $M$. A sequence of sections $s_{k}$ of $E_{k}$ is called asymptotically $J$-holomorphic if it satisfies the bounds $\left|\nabla^{r} s_{k}\right|_{g_{k}}=O(1)$ for $0 \leq r \leq 3$ and $\left|\nabla^{r-1} \bar{\partial} s_{k}\right|_{g_{k}}=O\left(k^{-1 / 2}\right)$ for $1 \leq r \leq 3$.

In this definition and throughout the text, the notation $O(1)$ means that there exists a bound by a uniform constant depending neither on the point of $M$ nor on the value of $k$.

If $\mathcal{L}$ is a Lagrangian submanifold of $M$, then $\left.[\omega]\right|_{\mathcal{L}}=0$ in $H^{2}(\mathcal{L}, \mathbb{R})$. Therefore $\left.h\right|_{\mathcal{L}}$ is torsion in $H^{2}(\mathcal{L}, \mathbb{Z})$ and there exists a positive integer $p$ such that $\left.p h\right|_{\mathcal{L}}=0$. We substitute the symplectic form $p \omega$ for $\omega$ on $M$, so that the first Chern class $c_{1}(L)$ becomes zero when restricted to $\mathcal{L}$. Therefore 
the line bundle $\left.L\right|_{\mathcal{L}}$ is topologically trivial, and the connection $\left.\nabla\right|_{\mathcal{L}}$ is flat. Since the holonomy of $\left.\nabla\right|_{\mathcal{L}}$ need not be trivial, we cannot expect to find a parallel section of $\left.L^{\otimes k}\right|_{\mathcal{L}}$. However, by choosing a suitable trivialization of $\left.L^{\otimes k}\right|_{\mathcal{L}}$, we can ensure that the connection 1 -form is bounded by a constant (w.r.t. the metric $g$ ), which gives the following result (Lemma 2 of $[6]$ ):

Lemma 2.2. The restriction of $L^{\otimes k}$ to $\mathcal{L}$ admits a section $\sigma_{\mathcal{L}, k}$ such that $\left|\sigma_{\mathcal{L}, k}\right|=1$ and $\left|\nabla \sigma_{\mathcal{L}, k}\right|_{g_{k}}=O\left(k^{-1 / 2}\right)$ at every point of $\mathcal{L}$.

Moreover, if we are given a fixed contractible open subset $U \subset \mathcal{L}$, then we can additionally assume that $\nabla \sigma_{\mathcal{L}, k}=0$ at every point of $U$. Let us fix sections $\sigma_{\mathcal{L}, k}$ satisfying all these properties once and for all.

We will also need the following lemma:

Lemma 2.3. Let $x \in M$. Then there exist Darboux coordinates with respect to the symplectic form $k \omega, \Psi_{k, x}: B_{g_{k}}\left(x, c k^{1 / 2}\right) \rightarrow \mathbb{C}^{n}$ (where $c>0$ is a fixed constant independent of $x$ and $k)$ such that: $(a) \Psi_{k, x}(x)=0 ;(b)\left(\Psi_{k, x}\right)_{*}$ identifies the complex structure $J_{x}$ of $T_{x} M$ with the standard complex structure $J_{0}$ on $\mathbb{C}^{n}$; and $(c) \Psi_{k, x}$ is approximately isometric, i.e., the map $\Psi_{k, x}$ satisfies $\left|\nabla^{r} \Psi_{k, x}\right|=O(1)$ for $r=1,2,3$ and $\left|\nabla^{r-1} \bar{\partial} \Psi_{k, x}(z)\right|=O\left(k^{-1 / 2}|z|\right)$ for $r=1,2,3$. Moreover, if $x \in \mathcal{L}$ we can take $\Psi_{k, x}$ to map the Lagrangian submanifold to $\mathbb{R}^{n}$.

Proof. The first part of this result is Lemma 3 in $[\mathbf{3}]$ (see also [9]). In the case where $x$ lies in the Lagrangian submanifold $\mathcal{L}$ of $M$, we use Weinstein neighborhood theorem to make the charts $\Psi_{k, x}$ map $\mathcal{L}$ to $\mathbb{R}^{n}$.

Note that the estimates in Lemmas 2.2 and 2.3 depend on the geometry of the submanifold $\mathcal{L}$ (in particular, its injectivity radius with respect to the metric $g$ ). With the aid of the fixed sections $\sigma_{\mathcal{L}, k}$, we can give the following definition.

Definition 2.4. An asymptotically holomorphic sequence $s_{k}$ of sections of $L^{\otimes k}$ on $M$ will be called real if

$$
\left.s_{k}\right|_{\mathcal{L}}=f_{k} \cdot \sigma_{\mathcal{L}, k},
$$

for some non-negative real function $f_{k}$.

For example, the sections constructed in Lemma 2.6 below are real. We are interested in this property because we will show later how to preserve it under the usual local perturbations in asymptotically holomorphic theory. In particular, we use from $[\boldsymbol{6}]$ the following.

Theorem 2.5. Given a symplectic manifold $(M, \omega)$ and a Lagrangian submanifold $\mathcal{L}$, there exists an asymptotically holomorphic sequence of sections $s_{k}: M \rightarrow L^{\otimes k}$ such that

(i) $\forall x \in \mathcal{L},\left|s_{k}(x)\right| \geq \eta$, for some $\eta>0$ independent of $k$. 
(ii) $\forall x \in M,\left|s_{k}(x)\right| \leq e^{-d_{k}(x, \mathcal{L})^{2} / 5}$.

(iii) $\forall x \in M$, such that $d_{k}(x, \mathcal{L}) \geq 2 k^{1 / 6}$, we have $s_{k}(x)=0$.

(iv) $s_{k}$ is real.

In order to prove this Theorem, we need the following.

Lemma 2.6. Let $x \in M$. There exists a sequence of asymptotically holomorphic sections $\sigma_{k, x}^{\text {ref }}$ satisfying the following estimates

- $\left|\sigma_{k, x}^{\mathrm{ref}}(y)\right| \geq \frac{1}{2}$ for all $y \in B_{g_{k}}(x, 1)$.

- $\left|\sigma_{k, x}^{\mathrm{ref}}(y)\right|+\left|\nabla \sigma_{k, x}^{\mathrm{ref}}(y)\right|+\left|\nabla^{2} \sigma_{k, x}^{\mathrm{ref}}(y)\right| \leq p\left(d_{k}(x, y)\right) e^{-d_{k}(x, y)^{2} / 5}$, where $p(t)$ is a fixed real polynomial.

- $\sigma_{k, x}^{\mathrm{ref}}$ has support in the ball $B_{g_{k}}\left(x, 2 k^{1 / 6}\right)$.

- If $x \in \mathcal{L}$, there is a real non-negative function $f_{k}: \mathcal{L} \rightarrow \mathbb{R}$ such that $\left.\sigma_{k, x}^{\mathrm{ref}}\right|_{\mathcal{L}}=f_{k} \cdot \sigma_{\mathcal{L}, k}$.

Proof. The existence of reference sections $\sigma_{k, x}^{\text {ref }}$ satisfying the first three properties follows from Lemma 3 in [2] (see also Proposition 11 in [9]). To get the last property, consider the Darboux chart $\Psi_{k, x}: B_{g_{k}}\left(x, c k^{1 / 2}\right) \rightarrow \mathbb{C}^{n}$ provided by Lemma 2.3 , which maps the Lagrangian submanifold to $\mathbb{R}^{n}$. Now we trivialize the positive line bundle $L^{\otimes k}$ in $B_{g_{k}}\left(x, c k^{1 / 2}\right)$ following radial directions. This trivialization yields a radially parallel local section $s_{r}^{k}$, such that $\left|\nabla\left(\sigma_{\mathcal{L}, k} /\left(s_{r}^{k} \mid \mathcal{L}\right)\right)\right|=O\left(k^{-1 / 2}\right)$ by Lemma 2.2 . The localized sections obtained in Lemma 3 in $[\mathbf{2}]$ are of the form

$$
\sigma_{k, x, 0}^{\mathrm{ref}}=e^{-|z|^{2} / 4} \chi(|z|) s_{r}^{k}
$$

where $\chi(x)$ is a real positive cut-off function which equals 1 in a ball of

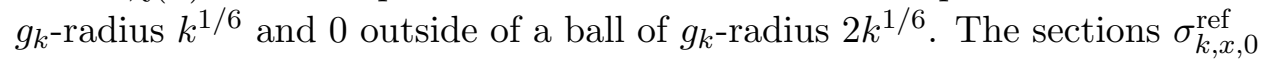
satisfy all desired properties except that over $\mathcal{L}$ they are real multiples of $s_{r}^{k}$ instead of $\sigma_{\mathcal{L}, k}$. However, we can choose a function $\phi: B_{g_{k}}\left(x, 2 k^{1 / 6}\right) \rightarrow \mathbb{R}$ such that (i) $|\phi|_{C^{2}}=O\left(k^{-1 / 2}\right)$ and (ii) $\sigma_{\mathcal{L}, k}=\left.e^{i \phi} s_{r}^{k}\right|_{\mathcal{L}}$. Then (i) implies that $\sigma_{k, x}^{\mathrm{ref}}=e^{i \phi} \sigma_{k, x, 0}^{\mathrm{ref}}$ still satisfies the first three properties, while (ii) implies that $\sigma_{k, x}^{\text {ref }}$ is a positive multiple of $\sigma_{\mathcal{L}, k}$ over $\mathcal{L}$.

Remark 2.7. Assume that there exists a neighborhood $U$ of $x$, containing $B_{g_{k}}\left(x, 2 k^{1 / 6}\right)$, over which $J$ is integrable and standard in local Darboux coordinates. If $x \in \mathcal{L}$, assume moreover that $\sigma_{\mathcal{L}, k}$ is covariantly constant over $U \cap \mathcal{L}$. Then $\sigma_{k, x}^{\text {ref }}$ is $J$-holomorphic over $B_{g_{k}}\left(x, k^{1 / 6}\right)$.

Let us now sketch a proof of Theorem 2.5.

Proof of Theorem 2.5. Take a set of points $S_{k}$ of $\mathcal{L}$ such that the balls $B_{g_{k}}(p, 1), p \in S_{k}$, cover $\mathcal{L}$ and any point of $\mathcal{L}$ belongs to at most $N$ such balls (where $N$ is a constant depending only on the dimension). Then we 
define a global section

$$
s_{k}=\sum_{x_{j} \in S_{k}} w_{j} \sigma_{k, x_{j}}^{\mathrm{ref}}
$$

where $\sigma_{k, x_{j}}^{\mathrm{ref}}$ are provided by Lemma 2.6 and $w_{j} \in \mathbb{C}$ are constants. If we choose $w_{j}=1$, then the sequence is asymptotically holomorphic and satisfies all the properties listed in the statement of the theorem.

We also have the usual notion of estimated transversality [2]:

Definition 2.8. A section $s_{k}$ of the bundle $E \otimes L^{\otimes k}$ is $\eta$-transverse to 0 if, for every $x \in M$ such that $\left|s_{k}(x)\right|<\eta, \nabla s_{k}(x)$ has a right inverse $\theta_{k}$ such that $\left|\theta_{k}\right|_{g_{k}}<\eta^{-1}$.

Lemma 2.9. Let $\mathcal{L}$ be a Lagrangian submanifold of $M$ and let $\varphi_{k}$ be an asymptotically holomorphic sequence of complex valued functions (i.e., sections of the trivial complex bundle) defined in some neighborhoods of $\mathcal{L}$. Suppose that $h_{k}=\left.\varphi_{k}\right|_{\mathcal{L}}$ is a map that factors through an immersion of a curve $C \hookrightarrow \mathbb{C}$. If $h_{k}$ satisfies the following property (identifying $\nabla \nabla h_{k}(x)$ with a linear map $T_{x} \mathcal{L} \rightarrow T_{x} \mathcal{L} \cong T_{x} \mathcal{L} \otimes T_{h_{k}(x)} C \subset T_{x} \otimes \mathbb{C}$ by means of the metric $\left.g_{k}\right)$ :

$$
\begin{aligned}
\left|\nabla h_{k}(x)\right|<\eta \Longrightarrow & \nabla \nabla h_{k}(x): T_{x} \mathcal{L} \rightarrow T_{x} \mathcal{L} \text { multiplies the length } \\
& \text { of vectors by at least } \eta
\end{aligned}
$$

for any $x \in \mathcal{L}$, then $\nabla \varphi_{k}$ is $\eta / 2$-transverse to 0 on a $g_{k}$-neighborhood of $\mathcal{L}$ of uniform radius.

Proof. For $x \in \mathcal{L}$, if $\left|\nabla \varphi_{k}(x)\right|<\eta$ then $\left|\nabla h_{k}(x)\right|<\eta$, hence $\nabla \nabla h_{k}(x)$ : $T_{x} \mathcal{L} \rightarrow T_{x} \mathcal{L}$ multiplies the length of vectors by at least $\eta$. But $T_{x} M=T_{x} \mathcal{L} \oplus$ $J\left(T_{x} \mathcal{L}\right)$ and the direct sum is an orthogonal one. Since the Hessian $\nabla \nabla \varphi_{k}(x)$ is approximately holomorphic, it equals the complexification of $\nabla \nabla h_{k}(x)$ up to some error of order $O\left(k^{-1 / 2}\right)$. Therefore, $\nabla \nabla \varphi_{k}(x)$ multiplies the length of vectors by at least $3 \eta / 4$. It can be checked that this property is equivalent to the $3 \eta / 4$-transversality of $\nabla \varphi_{k}$ in the sense of Definition 2.8. From this it follows the $\eta / 2$-transversality in a $g_{k}$-neighborhood of $\mathcal{L}$ of uniform radius.

Definition 2.10. Let $h_{k}: \mathcal{L} \rightarrow \mathbb{C}$ be a sequence of functions factoring through an immersion of a curve in $\mathbb{C}$. We say that $\nabla h_{k}$ is $\eta$-transverse if, for every $x \in \mathcal{L}$ such that $\left|\nabla h_{k}(x)\right|<\eta$, the map $\nabla \nabla h_{k}(x): T_{x} \mathcal{L} \rightarrow T_{x} \mathcal{L}$ multiplies the length of vectors by at least $\eta$.

Finally, recall the following result, due to Donaldson [10], which implies Theorem 1.2:

Proposition 2.11. Given an asymptotically holomorphic sequence of sections $s_{k}^{1} \oplus s_{k}^{2}$ of $L^{\otimes k} \oplus L^{\otimes k}$, and given $\delta>0$, there exists a sequence of 
sections $\sigma_{k}^{1} \oplus \sigma_{k}^{2}$ with $\left|\sigma_{k}^{j}-s_{k}^{j}\right|_{C^{2}} \leq \delta$, for $j=1,2$, satisfying the following properties:

(i) $\sigma_{k}^{1}$ is $\epsilon$-transverse to zero over $M$, for some uniform $\epsilon>0$.

(ii) $\sigma_{k}^{1} \oplus \sigma_{k}^{2}$ is $\epsilon$-transverse to zero over $M$.

(iii) Denoting by $Z_{k, \epsilon}=\left\{p \in M:\left|\sigma_{k}^{1}\right| \leq \epsilon\right\}$, the map $\partial\left(\sigma_{k}^{2} / \sigma_{k}^{1}\right)$ is $\epsilon$ transverse to zero in $M-Z_{k, \epsilon}$.

Moreover, after a small perturbation around the critical points we can ensure that $\phi_{k}=\sigma_{k}^{2} / \sigma_{k}^{1}$ is a symplectic Lefschetz pencil.

\section{Deformations of a Morse function}

The first step in our proof of Theorem 1.3 is to perturb the given Morse function $f$ on $\mathcal{L}$ to a suitable sequence of functions $h_{k}$. We need this sequence to satisfy that $\nabla h_{k}$ be transverse to zero in an estimated way. This is a necessary condition to have the functions $h_{k}$ arise as restrictions of Donaldson pencils (by a converse to Lemma 2.9). There are many ways of achieving such transversality, however we have to be careful because we will try to approximate $h_{k}$ by a combination of sections constructed by Lemma 2.6 that needs to be controlled in a precise way. This is the reason of the extra requirements that we will impose.

Let $f$ be a Morse function on $\mathcal{L}$. Consider a critical point $p$ of $f$, i.e., $\nabla f(p)=0$. Then for a suitable chart $\phi=\left(x_{1}, \ldots, x_{n}\right): U \rightarrow \mathbb{R}^{n}$ with $\phi(p)=0$ and which is isometric at $p$, we can write $f=c+\lambda_{1} x_{1}^{2}+\cdots+\lambda_{n} x_{n}^{2}$ for suitable non-zero real numbers $\lambda_{1}, \ldots, \lambda_{n}$. We start by deforming $f$ in a neighborhood of each critical point so that we can write $f=c+\epsilon_{1} x_{1}^{2}+\cdots+$ $\epsilon_{n} x_{n}^{2}$, where $\epsilon_{i}= \pm 1$ depending on whether $\lambda_{i}$ is positive or negative. We call $f$ again this new function. Moreover, for simplicity we also deform the almost complex structure $J$ in a neighborhood of every critical point so that it is integrable and standard in Darboux coordinates. Therefore, in such a neighborhood, $J$ and $g$ have a standard form. Let $r_{1}>0$ be a constant such that $f$ and $J$ can be written in such a standard way over the entire ball of radius $r_{1}$ around each critical point.

By Lemma 2.3 there exists some $r_{2}>0$ such that all Darboux charts $\Psi_{k, x}$ are "approximately isometric" in $B_{g}\left(x, r_{2}\right)$, i.e., the differential of $\Psi_{k, x}$ and its inverse distort the metric in a controlled manner at any point of the chart (in the sense of Lemma 2.3). Let $c_{0}$ be the minimum of $r_{1}$ and $r_{2}$. Note that for a critical point $p, \Psi_{k, p}$ is actually holomorphic and an isometry, since $J$ and $g$ are standard in $B_{g_{k}}\left(p, c_{0} k^{1 / 2}\right)$.

We need the following technical Lemma that will be used to define deformations of a Morse function. 
Lemma 3.1. Let $l: \mathbb{R}_{+} \rightarrow \mathbb{R}_{+}$be a smooth function with $l^{\prime}(t)<0$ and $l^{\prime}(t) / l(t) \geq-(3 / 4 t)$ for all $t>0$. Then the smooth function $F: \mathbb{R}^{n}-\{0\} \rightarrow$ $\mathbb{R}^{n}-\{0\}$ defined by $F(x)=l(|x|) \cdot x$ satisfies the following properties:

(i) $d F(x)=l(|x|)\left(\left(d x_{1}, \ldots, d x_{n}\right)+\frac{l^{\prime}(|x|)}{l(|x|)|x|}\left(x_{1} x_{1} d x_{1}+\cdots+x_{1} x_{n} d x_{n}, \ldots\right.\right.$, $\left.\left.x_{n} x_{1} d x_{1}+\cdots+x_{n} x_{n} d x_{n}\right)\right)$, i.e., using matrix notation, $d F(x)=$ $l(|x|) \operatorname{Id}+\frac{l^{\prime}(|x|)}{|x|}\left(x \otimes x^{t}\right)$.

(ii) $\operatorname{det}(d F(x))=l(|x|)^{n}\left(1+\frac{|x| l^{\prime}(|x|)}{l(|x|)}\right) \geq \frac{l(|x|)^{n}}{4}$.

(iii) $|d F(x)| \leq l(|x|)$.

(iv) The minimum eigenvalue of $d F(x)$ is greater or equal to $l(|x|) / 4$.

Proof. The first property is a simple computation. The next three properties follow from the expression for the linear map $d F(x)$, which can be diagonalized by means of an orthogonal transformation mapping $x$ to the first coordinate axis. It is then clear that the eigenvalues of $d F(x)$ are $l(|x|)$ with multiplicity $n-1$, and $l(|x|)+l^{\prime}(|x|)|x|$ with multiplicity 1 .

Proposition 3.2. Let $f: \mathcal{L} \rightarrow[0,1]$ be a Morse function and let $D>0$ be a large enough constant. Then there exists a sequence $h_{k}: \mathcal{L} \rightarrow\left[0, k^{1 / 2}\right]$ of deformations of $f$ satisfying (with respect to the metric $g_{k}$ over $\mathcal{L}$ )

(i) $\left|\nabla h_{k}\right| \leq C_{0} D,\left|\nabla \nabla h_{k}\right| \leq C_{0}$,

(ii) $\nabla h_{k}$ is $\eta$-transverse to zero, for some $\eta>0$ independent of $k$ and $D$, (iii) $\left|\nabla^{3} h_{k}\right| \leq C_{0} / D$,

where $C_{0}$ is a constant independent of $k$ and $D$.

To prove the main result, we will need to choose a sufficiently large value of the constant $D$; this requires a slightly more complicated argument (otherwise one could set $\epsilon=0$ in the formulas below).

Proof. Denote by $\left\{p_{j}\right\}$ the set of critical points of $f$. We are assuming that $f$ is of the form

$$
f\left(x_{1}, \ldots, x_{n}\right)=c_{j}+\sum \pm x_{i}^{2}
$$

in a neighborhood $B_{g}\left(p_{j}, c_{0}\right)$ of $p_{j}$. Scaling the coordinates we get that, over $B_{g_{k}}\left(p_{j}, c_{0} k^{1 / 2}\right), f$ is expressed as

$$
f\left(x_{1}, \ldots, x_{n}\right)=c_{j}+\frac{1}{k} \sum \pm x_{i}^{2},
$$

in the chart $\Psi_{k, p_{j}}$. These new coordinates are actually isometric over the ball $B_{g_{k}}\left(p_{j}, c_{0} k^{1 / 2}\right)$, because of the choice of almost-complex structure $J$ made at the beginning of this section. Now define the sequence $f_{k}=k^{1 / 2} f$. 
The derivatives of $f_{k}$ are

$$
\begin{aligned}
f_{k}\left(x_{1}, \ldots, x_{n}\right) & =k^{1 / 2} c_{j}+\frac{1}{k^{1 / 2}} \sum\left( \pm x_{i}^{2}\right), \\
\nabla f_{k}\left(x_{1}, \ldots, x_{n}\right) & =\frac{1}{k^{1 / 2}} \sum\left( \pm 2 x_{i} d x_{i}\right), \\
\nabla \nabla f_{k}\left(x_{1}, \ldots, x_{n}\right) & =\frac{2}{k^{1 / 2}} \sum\left( \pm d x_{i} \otimes d x_{i}\right) .
\end{aligned}
$$

So $f_{k}$ satisfies, without any perturbation,

$$
\begin{aligned}
\left|\nabla f_{k}\right|_{g_{k}} & =O(1) \\
\left|\nabla \nabla f_{k}\right|_{g_{k}} & =O\left(k^{-1 / 2}\right) .
\end{aligned}
$$

Now take a cut-off function $l_{k}:\left[0, k^{1 / 2} c_{0}\right] \rightarrow\left[0, k^{1 / 4}\right]$ satisfying the following properties:

- $l_{k}(t)=k^{1 / 4}$, for $t \in[0, D]$.

- $l_{k}(t)=\left(a k^{1 / 4} / t^{1 / 2+\epsilon}\right)$, for $t \in\left[2 D, k^{1 / 2} c_{0} / 2\right]$, where $a$ and $\epsilon$ are positive constants to be adjusted below ( $a$ converges to a fixed positive value as $k \rightarrow \infty$, while $\epsilon=\epsilon(k) \rightarrow 0$ as $k \rightarrow \infty)$.

- $l_{k}(t)=1$, for $t>3 k^{1 / 2} c_{0} / 4$.

- In the interval $[D, 2 D], l_{k}$ is decreasing, $\left|l_{k}^{\prime}(t)\right| \leq \epsilon_{2} k^{1 / 4} / D$ and $\left|l_{k}^{\prime \prime}(t)\right| \leq$ $C k^{1 / 4} / D^{2},\left|l_{k}^{\prime \prime \prime}(t)\right| \leq C k^{1 / 4} / D^{3}$ for constants $\epsilon_{2}, C$ independent of $k$.

- In the interval $\left[k^{1 / 2} c_{0} / 2,3 k^{1 / 2} c_{0} / 4\right], l_{k}$ is decreasing, $\left|l_{k}^{\prime}(t)\right| \leq \epsilon_{2}^{\prime} k^{-1 / 2}$ and $\left|l_{k}^{\prime \prime}(t)\right| \leq C^{\prime} k^{-1},\left|l_{k}^{\prime \prime \prime}(t)\right| \leq C^{\prime} k^{-3 / 2}$ for constants $\epsilon_{2}^{\prime}, C^{\prime}$ independent of $k$.

- The following inequality holds over $\left(D, 3 k^{1 / 2} c_{0} / 4\right)$ :

$$
0>\frac{l_{k}^{\prime}(t)}{l_{k}(t)} \geq-\frac{1 / 2+\epsilon}{t}
$$

We adjust $a$ and $\epsilon$ in the following way: the function $\left(a k^{1 / 4} / t^{1 / 2+\epsilon}\right)$ equals $k^{1 / 4}$ for $t=(3 / 2) D$ and equals 1 for $t=0.7 c_{0} k^{1 / 2}$. Therefore

$$
\begin{aligned}
a & =\left(\frac{3}{2} D\right)^{1 / 2+\epsilon} \\
\epsilon & =\frac{\ln \left(3 D / 1.4 c_{0}\right)}{\ln k-2 \ln \left(3 D / 1.4 c_{0}\right)}
\end{aligned}
$$

The inequality $k \geq\left(3 D / 1.4 c_{0}\right)^{6}$ must hold in order to have $0<\epsilon \leq(1 / 4)$. With these choices of $a$ and $\epsilon$, it is easy to find a function $l_{k}(t)$ with the desired properties; observe in particular that (3.3) automatically holds when $l_{k}$ is of the form $l_{k}(t)=G(t) \cdot\left(a k^{1 / 4} / t^{1 / 2+\epsilon}\right)$ for some increasing function $G$ (by differentiating the identity $\log l_{k}=\log G-\left(\frac{1}{2}+\epsilon\right) \log t+C$ ).

The following figure gives the shape of $l_{k}(t)$ : 


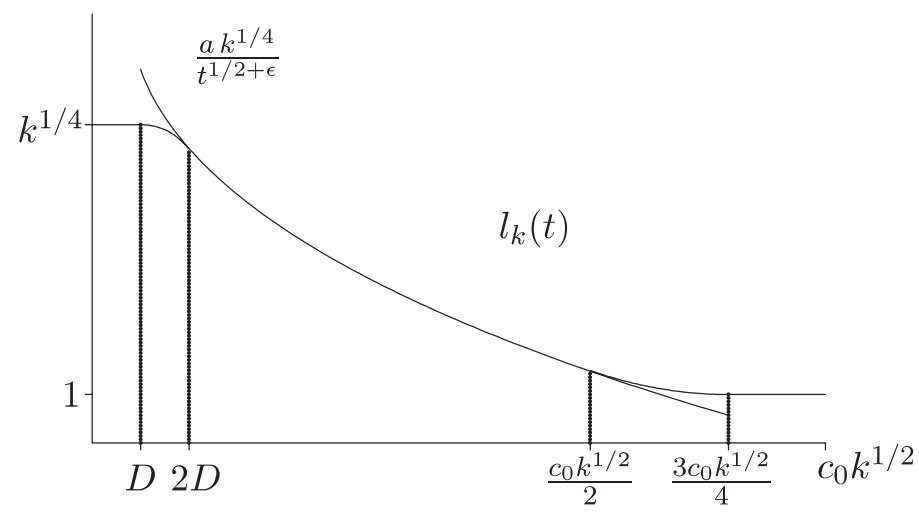

Note that $l_{k}(t) \cdot t$ is an increasing function, and moreover it is a diffeomorphism $l_{k}(t) \cdot t:(0, \infty) \rightarrow(0, \infty)$.

Now we define the function $\hat{l}_{k}\left(x_{1}, \ldots, x_{n}\right)=l_{k}\left(\left|\left(x_{1}, \ldots, x_{n}\right)\right|\right)$ in a small neighborhood of the critical points. This implies the following formulae for $\hat{l}_{k}$ :

$$
\begin{aligned}
& \left|\nabla \hat{l}_{k}(x)\right| \leq C_{1}\left|l_{k}^{\prime}\right| \leq C_{1} \frac{\left|l_{k}\right|}{|x|} \\
& \left|\nabla^{2} \hat{l}_{k}(x)\right| \leq C\left|l_{k}^{\prime \prime}\right|+C \frac{\left|l_{k}^{\prime}\right|}{|x|} \leq C_{2} \frac{\left|l_{k}\right|}{|x|^{2}} \\
& \left|\nabla^{3} \hat{l}_{k}(x)\right| \leq C\left|l_{k}^{\prime \prime \prime}\right|+C \frac{\left|l_{k}^{\prime \prime}\right|}{|x|}+C \frac{\left|l_{k}^{\prime}\right|}{|x|^{2}} \leq C_{3} \frac{\left|l_{k}\right|}{|x|^{3}}
\end{aligned}
$$

for some uniform constants $C_{1}, C_{2}, C_{3}$. These are simple computations in the middle interval and in $[D, 2 D]$ and $\left[k^{1 / 2} c_{0} / 2,3 k^{1 / 2} c_{0} / 4\right]$, using the bounds on the derivatives of $l_{k}$.

We construct a perturbation of $f$ by setting:

$$
h_{k}(x)= \begin{cases}f_{k}\left(\hat{l}_{k}(x) \cdot x\right), & \forall x \in \bigcup_{j} B_{g_{k}}\left(p_{j}, k^{1 / 2} c_{0}\right) \\ f_{k}(x), & \text { otherwise. }\end{cases}
$$

This function is isotopic to the original $f_{k}$, since $\hat{l}_{k}(x) \cdot x: B_{g_{k}}\left(p_{j}, k^{1 / 2} c_{0}\right) \rightarrow$ $B_{g_{k}}\left(p_{j}, k^{1 / 2} c_{0}\right)$ is a diffeomorphism isotopic to the identity (relative to the boundary).

Outside the union of the balls $B_{g_{k}}\left(p_{j}, 3 k^{1 / 2} c_{0} / 4\right)$, the estimates (3.2) imply that $h_{k}$ satisfies (i). Moreover, in that region, there is a constant $\xi>0$ independent of $k$ such that $\left|\nabla h_{k}\right|_{g_{k}} \geq \xi$, which implies that $\nabla h_{k}$ is $\xi$-transverse to zero, so (ii) also holds.

In the ball $B_{g_{k}}\left(p_{j}, D\right)$, we have $h_{k}=k^{1 / 2} c_{j}+\sum\left( \pm x_{i}^{2}\right)$, so $h_{k}$ satisfies (i) and (ii). 
Now let us see what happens in the annuli $B_{g_{k}}\left(p_{j}, 3 k^{1 / 2} c_{0} / 4\right)-B_{g_{k}}\left(p_{j}, D\right)$. We start by computing the derivatives of $h_{k}$,

$$
\begin{aligned}
\nabla h_{k}(x)= & \nabla f_{k}\left(\hat{l}_{k}(x) \cdot x\right) \circ\left(d \hat{l}_{k}(x) \cdot x+\hat{l}_{k}(x) \cdot \mathrm{Id}\right) \\
\nabla \nabla h_{k}(x)= & \nabla \nabla f_{k}\left(\hat{l}_{k}(x) \cdot x\right) \circ\left(( d \hat { l } _ { k } ( x ) \cdot x + \hat { l } _ { k } ( x ) \cdot \mathrm { Id } ) \otimes \left(d \hat{l}_{k}(x) \cdot x\right.\right. \\
& \left.\left.+\hat{l}_{k}(x) \cdot \mathrm{Id}\right)\right)+\nabla f_{k}\left(\hat{l}_{k}(x) \cdot x\right)\left(\nabla \nabla \hat{l}_{k}(x) \cdot x\right. \\
& \left.+d \hat{l}_{k}(x) \otimes \mathrm{Id}+\mathrm{Id} \otimes d \hat{l}_{k}(x)\right) .
\end{aligned}
$$

Note that we have, using (3.1),

$$
\left|\nabla f_{k}\left(\hat{l}_{k}(x) \cdot x\right)\right|=\frac{2}{k^{1 / 2}}\left|\hat{l}_{k}(x) \cdot x\right|=\frac{2}{k^{1 / 2}} l_{k}(|x|)|x| .
$$

Also, consider $d\left(\hat{l}_{k}(x) \cdot x\right)=d \hat{l}_{k}(x) \cdot x+\hat{l}_{k}(x) \cdot$ Id. The function $l_{k}$ satisfies that $l_{k}^{\prime}(t)<0$ and $\left(l_{k}^{\prime}(t) / l_{k}(t)\right) \geq-(3 / 4 t)$. Hence we may apply Lemma 3.1 to get that the eigenvalues of $d\left(\hat{l}_{k}(x) \cdot x\right)$ are in the range $\left[(1 / 4) l_{k}(|x|), l_{k}(|x|)\right]$.

By construction, we have, in the region $D \leq t \leq 3 c_{0} k^{1 / 2} / 4$, that

$$
\frac{c_{1} D^{1 / 2+\epsilon} k^{1 / 4}}{t^{1 / 2+\epsilon}} \leq l_{k}(t) \leq \frac{c_{2} D^{1 / 2+\epsilon} k^{1 / 4}}{t^{1 / 2+\epsilon}},
$$

for some positive constants $c_{1}, c_{2}$ independent of $k$ and $D$. Therefore equation (3.5) implies

$$
\left|\nabla h_{k}(x)\right| \leq \frac{2}{k^{1 / 2}} l_{k}(|x|)|x| l_{k}(|x|) \leq \frac{C D^{1+2 \epsilon}}{|x|^{2 \epsilon}} \leq C_{0} D
$$

for some uniform $C_{0}>0$. Analogously,

$$
\left|\nabla h_{k}(x)\right| \geq \frac{c D^{1+2 \epsilon}}{|x|^{2 \epsilon}} \geq \frac{c D^{1+2 \epsilon}}{\left(c_{0} k^{1 / 2}\right)^{2 \epsilon}} \geq \xi
$$

for some uniform $\xi$, thus proving (ii). To finish proving (i), we have to bound the second derivative.

$$
\begin{aligned}
\left|\nabla \nabla h_{k}(x)\right|= & \mid \nabla \nabla f_{k}\left(\hat{l}_{k}(x) \cdot x\right) \circ\left(( d \hat { l } _ { k } ( x ) \cdot x + \hat { l } _ { k } ( x ) \cdot \mathrm { Id } ) \otimes \left(d \hat{l}_{k}(x) \cdot x\right.\right. \\
& \left.\left.+\hat{l}_{k}(x) \cdot \mathrm{Id}\right)\right)+\nabla f_{k}\left(\hat{l}_{k}(x) \cdot x\right)\left(\nabla \nabla \hat{l}_{k}(x) \cdot x\right. \\
& \left.+d \hat{l}_{k}(x) \otimes \mathrm{Id}+\mathrm{Id} \otimes d \hat{l}_{k}(x)\right) \mid \\
\leq & \frac{2}{k^{1 / 2}} l_{k}(|x|)^{2}+\frac{2}{k^{1 / 2}} l_{k}(|x|)|x|\left[C_{2} \frac{l_{k}(|x|)}{|x|}+2\left|l_{k}^{\prime}(|x|)\right|\right] \\
\leq & C \frac{l_{k}(|x|)^{2}}{k^{1 / 2}} \leq C_{0},
\end{aligned}
$$

using equation (3.4) and $\left|l_{k}^{\prime}\right||x| \leq(3 / 4) l_{k}$.

Finally we prove (iii) by considering the third derivative of $h_{k}$. If the considered point is not close to a critical point then $f_{k}$ has not been perturbed, 
i.e., $h_{k}=f_{k}$ and we have $\left|\nabla \nabla \nabla f_{k}\right|_{g_{k}}=O\left(k^{-1}\right)$. To bound $\nabla \nabla \nabla h_{k}$ in a ball $B_{g_{k}}\left(p_{j}, c_{0} k^{1 / 2}\right)$ around a critical point $p_{j}$, we compute from equation (3.6),

$$
\begin{aligned}
\left|\nabla \nabla \nabla h_{k}\right|= & \mid \sum^{\prime} \nabla \nabla f_{k}\left(\hat{l}_{k}(x) \cdot x\right) \circ\left(\left(d \hat{l}_{k}(x) \cdot x+\hat{l}_{k}(x) \cdot \mathrm{Id}\right)\right. \\
& \left.\otimes\left(\nabla \nabla \hat{l}_{k}(x) \cdot x+d \hat{l}_{k}(x) \otimes \mathrm{Id}+\mathrm{Id} \otimes d \hat{l}_{k}(x)\right)\right) \\
& +\nabla f_{k}\left(\hat{l}_{k}(x) \cdot x\right) \circ\left(\nabla \nabla \nabla \hat{l}_{k}(x) \cdot x+\sum^{\prime} \nabla \nabla \hat{l}_{k}(x) \otimes \mathrm{Id}\right) \mid \\
\leq & 3 \frac{2}{k^{1 / 2}} l_{k}(|x|)\left(C_{2}+2\right) \frac{l_{k}(|x|)}{|x|}+\frac{2|x| l_{k}(|x|)}{k^{1 / 2}}\left(C_{3}+3 C_{2}\right) \frac{l_{k}(|x|)}{|x|^{2}} \\
\leq & C \frac{l_{k}(|x|)^{2}}{k^{1 / 2}|x|} \leq \frac{C_{0}}{D},
\end{aligned}
$$

where $\sum^{\prime}$ denotes the sum of three terms obtained by cyclic permutation of the three factors in $T^{*} M \otimes T^{*} M \otimes T^{*} M$. This estimate completes the proof of the Proposition.

The purpose of the bound on $\left|\nabla^{3} h_{k}\right|$ given in Proposition 3.2 is to let us control the error produced by replacing $h_{k}$ by its order two Taylor approximation at a given point. Fix any point $x \in \mathcal{L}$ and consider the approximately isometric chart $\Psi_{k, x}$ of Lemma 2.3. Let $P_{2, x}$ be the degree 2 Taylor polynomial of $h_{k}$ at $x$ in these coordinates. Then we have in particular $\left|h_{k}(y)-P_{2, x}(y)\right| \leq\left|\nabla \nabla \nabla h_{k}(\xi)\right||y|^{3}$, where $\xi$ is a point of the segment joining 0 and $y$; hence, it follows from Proposition 3.2 (iii) that

$$
\left|\nabla^{i}\left(h_{k}(y)-P_{2, x}(y)\right)\right| \leq \frac{C}{D}|y-x|^{3-i}, \quad \text { for } y \in \mathcal{L} \cap B_{g_{k}}\left(x, k^{1 / 2} c_{0}\right), \quad i=0,1,2 .
$$

\section{Transversality in a neighborhood of the Lagrangian}

The objective now is to define two asymptotically holomorphic sections $s_{k}^{1}$ and $s_{k}^{2}$ whose quotient approximates $h_{k}$. This cannot be done directly because $\left|h_{k}\right|$ is not $O(1)$ and therefore if we take the sequence $s_{k}^{1}$ as given in Theorem 2.5, then $s_{k}^{2}=h_{k} \cdot s_{k}^{1}$ will not be bounded. We shall work in a different way.

Lemma 4.1. Let $h_{k}$ be a sequence of functions satisfying the bounds of Proposition 3.2. Define the sequences of functions $h_{k}^{1}$ and $h_{k}^{2}$ by

$$
\begin{aligned}
\left(h_{k}^{1}, h_{k}^{2}\right): \mathcal{L} & \longrightarrow S^{1} \\
x & \longrightarrow\left(\cos \left(h_{k}(x)\right), \sin \left(h_{k}(x)\right)\right) .
\end{aligned}
$$

Then these functions satisfy:

(i) $\left|h_{k}^{j}\right|=O(1)$, and $\left|\nabla^{r} h_{k}^{j}\right| \leq C_{0} D, r=1,2, j=1,2$, where $C_{0}>0$ is a constant independent of $k$ and $D$.

(ii) $\nabla\left(h_{k}^{1}, h_{k}^{2}\right)$ is $\eta$-transverse to zero, for some $\eta>0$ independent of $k$. 
(iii) Fix any point $x \in \mathcal{L}$ and consider the chart $\Psi_{k, x}$ of Lemma 2.3. Let $P_{2, x}$ be the degree two Taylor polynomial approximating $h_{k}$ at $x$. Then

- $\left|\nabla^{i}\left(h_{k}^{1}(y)-\cos \left(P_{2, x}(y)\right)\right)\right| \leq \frac{C}{D}|y-x|^{3-i}$, for $y \in \mathcal{L} \cap B_{g_{k}}\left(x, k^{1 / 2} c_{0}\right)$, $i=0,1,2$.

- $\left|\nabla^{i}\left(h_{k}^{2}(y)-\sin \left(P_{2, x}(y)\right)\right)\right| \leq \frac{C}{D}|y-x|^{3-i}$, for $y \in \mathcal{L} \cap B_{g_{k}}\left(x, k^{1 / 2} c_{0}\right)$, $i=0,1,2$.

Proof. The three properties are simple to check by using the chain rule and the Taylor approximation theorem.

Proposition 4.2. Fix a sequence of functions $h_{k}: \mathcal{L} \rightarrow \mathbb{R}$ satisfying the bounds of Proposition 3.2. Then for any $\delta>0$ there are two real asymptotically holomorphic sections $s_{k}^{1}$ and $s_{k}^{2}$ of $L^{\otimes k}$, supported over a fixed neighborhood of $\mathcal{L}$ in $M$, and such that

$$
\left.\left|\left(\cos \left(2 h_{k}\right), \sin \left(2 h_{k}\right)\right)-\left(\frac{s_{k}^{1}+i s_{k}^{2}}{s_{k}^{1}-i s_{k}^{2}}\right)\right|_{\mathcal{L}}\right|_{C^{2}} \leq \delta .
$$

The reason why we need a $C^{2}$ approximation is to make sure that the restriction to $\mathcal{L}$ of the pencil that we construct remains a Morse function isotopic to $h_{k}$.

Proof. Construct the associated sequence $\left(h_{k}^{1}, h_{k}^{2}\right)=\left(\cos \left(h_{k}\right), \sin \left(h_{k}\right)\right)$ which satisfies the bounds of Lemma 4.1. Let $\gamma>0$. Assume for a moment that we have two asymptotically holomorphic sections $s_{k}^{0}$ and $s_{k}^{1}$ defined over $M$ whose quotient approximates $h_{k}^{1}$ with error

$$
\left.\left|h_{k}^{1}-\left(\frac{s_{k}^{1}}{s_{k}^{0}}\right)\right|_{\mathcal{L}}\right|_{C^{2}} \leq \gamma
$$

Analogously, assume that we have $s_{k}^{0}$ and $s_{k}^{2}$ whose quotient approximates $h_{k}^{2}$ with error

$$
\left.\left|h_{k}^{2}-\left(\frac{s_{k}^{2}}{s_{k}^{0}}\right)\right|_{\mathcal{L}}\right|_{C^{2}} \leq \gamma
$$

Assume moreover that $\left.\left(s_{k}^{2} / s_{k}^{1}\right)\right|_{\mathcal{L}} \in \mathbb{R} \mathbb{P}^{1}=\mathbb{R} \cup\{\infty\}$. Now note that

and therefore

$$
\frac{h_{k}^{1}+i h_{k}^{2}}{h_{k}^{1}-i h_{k}^{2}}=\left(h_{k}^{1}+i h_{k}^{2}\right)^{2}=\left(\cos \left(2 h_{k}\right), \sin \left(2 h_{k}\right)\right)
$$

$$
\left.\left|\left(\cos \left(2 h_{k}\right), \sin \left(2 h_{k}\right)\right)-\left(\frac{s_{k}^{1}+i s_{k}^{2}}{s_{k}^{1}-i s_{k}^{2}}\right)\right|_{\mathcal{L}}\right|_{C^{2}} \leq \delta,
$$

if $\gamma>0$ is chosen small enough, depending on $\delta$. Note that since $\left.\left(s_{k}^{2} / s_{k}^{1}\right)\right|_{\mathcal{L}} \in$ $\mathbb{R P}^{1}$, the term $\left.\left(\left(s_{k}^{1}+i s_{k}^{2}\right) /\left(s_{k}^{1}-i s_{k}^{2}\right)\right)\right|_{\mathcal{L}}$ takes values on the unit circle.

To conclude we just have to find asymptotically holomorphic real sequences $s_{k}^{0}, s_{k}^{1}$ and $s_{k}^{2}$ satisfying equations (4.1) and (4.2). The condition of being 
real will ensure that $\left.\left(s_{k}^{2} / s_{k}^{1}\right)\right|_{\mathcal{L}} \in \mathbb{R P}^{1}$. Let us treat the case of $h_{k}^{1}$, the other one being analogous.

Let $s_{k}^{0}$ be a sequence of asymptotically holomorphic real sections as given by Theorem 2.5. Recall from Lemma 2.2 that we have a trivializing section $\sigma_{\mathcal{L}, k}$ of $\left.L^{\otimes k}\right|_{\mathcal{L}}$. There exists a set of points $\left\{x_{j}\right\}_{j \in J}$ in $\mathcal{L}$ such that the sequence of sections is

$$
s_{k}^{0}=\sum_{j \in J} w_{j} \sigma_{k, x_{j}}^{\mathrm{ref}}
$$

where $\sigma_{k, x_{j}}^{\text {ref }}$ are the local sections given by Lemma 2.6 and $w_{j}=1$. Remember that the points $x_{j}$ are chosen in such a way that the balls $B_{g_{k}}\left(x_{j}, 1\right)$ cover $\mathcal{L}$ and any point of $\mathcal{L}$ belongs to at most a fixed number of such balls. Recall that $s_{k}^{0}$ is real, and that $\left|s_{k}^{0}\right|_{\mathcal{L}} \mid \geq \eta$, for some constant $\eta>0$ independent of $k$.

For any given point $x_{j}$, take an approximately holomorphic chart $\Psi_{k, x_{j}}=$ $\left(z_{1}, \ldots, z_{n}\right)$ given by Lemma 2.3. This chart maps the Lagrangian submanifold to $\mathbb{R}^{n} \subset \mathbb{R}^{n} \oplus i \mathbb{R}^{n}=\mathbb{C}^{n}$. We consider the second order Taylor expansion of $h_{k}$ at $p_{j}$, which we denote by $P_{2, j}$. This Taylor expansion can be understood as a real degree 2 polynomial in the real parts of the Darboux coordinates, but $P_{2, j}$ can also be viewed as a complex polynomial (with real coefficients) in the whole $\mathbb{C}^{n}$. Recall that by Lemma 4.1 we have

$$
\left|\nabla^{i}\left(h_{k}^{1}(y)-\cos \left(P_{2, j}(y)\right)\right)\right| \leq \frac{C}{D}\left|y-x_{j}\right|^{3-i} \text {. }
$$

We define the section

$$
s_{k}^{1}=\sum_{x_{j} \in S} w_{j} \cos \left(P_{2, j}\right) \sigma_{k, x_{j}}^{\mathrm{ref}},
$$

and use $s_{k}^{1} / s_{k}^{0}$ as a candidate to approximate our sequence of functions $h_{k}^{1}$. It is easy to check that $s_{k}^{1}$ is real (since the sections $\sigma_{k, x_{j}}^{\text {ref }}$ and the polynomials $P_{2, j}$ are real); however, the existence of a uniform bound on $s_{k}^{1}$ depends on the growth of the function $\cos \left(P_{2, j}\right)$ away from $x_{j}$. For this purpose, note that we may assume from the beginning that $\left|\nabla \nabla h_{k}\right|$ is very small, say $\left|\nabla \nabla h_{k}\right|<\frac{1}{10}$, by multiplying $h_{k}$ by a small positive real constant (independent of $k$ and $D$ ), using the estimate of Proposition 3.2. Therefore for $x \in M$, we have

$$
\left|\cos \left(P_{2, j}(x)\right)\right| \leq e^{C_{0} D d_{k}\left(x, x_{j}\right)+d_{k}\left(x, x_{j}\right)^{2} / 10}
$$

and hence by Lemma 2.6 ,

$$
\left|\cos \left(P_{2, j}(x)\right) \sigma_{k, x_{j}}^{\mathrm{ref}}(x)\right| \leq p\left(d_{k}\left(x, x_{j}\right)\right) e^{C_{0} D d_{k}\left(x, x_{j}\right)-d_{k}\left(x, x_{j}\right)^{2} / 10} .
$$

From this it easily follows that $s_{k}^{1}$ is asymptotically holomorphic (with asymptotically holomorphic bounds depending on $D$, but this does not matter). Notice that this loss in the exponential decay only happens close 
to the critical points, since away from them the degree 2 term of $P_{2, j}$ is $O\left(k^{-1 / 2}\right)$ and so for large $k$ the extra factor $\cos \left(P_{2, j}\right)$ does not affect the decay properties of the product.

The $C^{0}$ error made with the approximation $s_{k}^{1} / s_{k}^{0}$ is

$$
\begin{aligned}
\left|h_{k}^{1}-\left(\frac{s_{k}^{1}}{s_{k}^{0}}\right)\right| \mathcal{L} \mid & =\frac{1}{\left|s_{k}^{0}\right|}\left|h_{k}^{1} \cdot s_{k}^{0}-s_{k}^{1}\right| \leq \frac{1}{\eta} \sum_{x_{j} \in S}\left|w_{j}\left(h_{k}^{1}-\cos \left(P_{2, j}\right)\right) \sigma_{k, x_{j}}^{\mathrm{ref}}\right| \\
& \leq \frac{C}{\eta D} \sum_{x_{j} \in S} d_{k}\left(\cdot, x_{j}\right)^{3}\left|\sigma_{k, x_{j}}^{\mathrm{ref}}\right| \leq \frac{C^{\prime}}{D}
\end{aligned}
$$

where $\eta$ is the constant provided by Theorem 2.5. Now let us check the $C^{1}$-norm of the error

$$
\begin{aligned}
\left|d h_{k}^{1}-d\left(\frac{s_{k}^{1}}{s_{k}^{0}}\right)\right|= & \left|d h_{k}^{1}-\frac{\nabla s_{k}^{1} \cdot s_{k}^{0}-s_{k}^{1} \cdot \nabla s_{k}^{0}}{\left(s_{k}^{0}\right)^{2}}\right| \\
= & \left|\frac{1}{s_{k}^{0}}\left(d h_{k}^{1} \cdot s_{k}^{0}-\nabla s_{k}^{1}+\frac{s_{k}^{1}}{s_{k}^{0}} \cdot \nabla s_{k}^{0}\right)\right| \\
\leq & \frac{1}{\eta}\left(\sum\left|w_{j}\right|\left|d h_{k}^{1}-d \cos \left(P_{2, j}\right)\right|\left|\sigma_{k, x_{j}}^{\mathrm{ref}}\right|\right. \\
& \left.+\sum\left|w_{j}\right|\left|h_{k}^{1}-\cos \left(P_{2, j}\right)\right|\left|\nabla \sigma_{k, x_{j}}^{\mathrm{ref}}\right|\left|\frac{s_{k}^{1}}{s_{k}^{0}}-h_{k}^{1}\right|\left|\nabla s_{k}^{0}\right|\right) \\
\leq & \frac{1}{\eta} \frac{C}{D}=\frac{C^{\prime}}{D} .
\end{aligned}
$$

Analogously, one can work out the $C^{2}$-norm of the error. Choosing $D$ large, one can make this smaller than any $\gamma>0$.

\section{Global transversality}

In order to prove Theorem 1.3, we use Proposition 3.2 to construct a sequence of maps $h_{k}: \mathcal{L} \rightarrow \mathbb{R}$. Then we use Proposition 4.2 to construct two sequences of real asymptotically holomorphic sections $s_{k}^{1}$ and $s_{k}^{2}$ of $L^{\otimes k}$ such that

$$
\frac{\sigma_{k}^{2}}{\sigma_{k}^{1}}=\left.\left(\frac{s_{k}^{1}+i s_{k}^{2}}{s_{k}^{1}-i s_{k}^{2}}\right)\right|_{\mathcal{L}}
$$

is a Morse function isotopic to $\left(\cos \left(2 h_{k}\right), \sin \left(2 h_{k}\right)\right)$. Here $\sigma_{k}^{1}=s_{k}^{1}-i s_{k}^{2}$ and $\sigma_{k}^{2}=s_{k}^{1}+i s_{k}^{2}$. Let us check the three transversality properties of Proposition 2.11 for this sequence in a tubular neighborhood of fixed $g_{k}$-radius of $\mathcal{L}$.

By the property given in the proof of Proposition 4.2,

$$
\left.\left|\left(h_{k}^{1}+i h_{k}^{2}\right)-\left(\frac{s_{k}^{1}+i s_{k}^{2}}{s_{k}^{0}}\right)\right|_{\mathcal{L}}\right|_{C^{2}}
$$


is small. Therefore $\left|\sigma_{k}^{2}\right|=\left|s_{k}^{1}+i s_{k}^{2}\right| \geq\left|s_{k}^{0}\right| / 2$ along $\mathcal{L}$. In particular $\sigma_{k}^{2}$ is transverse to zero along $\mathcal{L}$. Analogously, $\sigma_{k}^{1}$ and $\sigma_{k}^{1} \oplus \sigma_{k}^{2}$ are transverse to zero along $\mathcal{L}$. Thus the first two properties of Proposition 2.11 hold in a tubular neighborhood of $\mathcal{L}$ of some uniform $g_{k}$-radius. Moreover, the sequence $\nabla\left(h_{k}^{1}, h_{k}^{2}\right)$ is transverse to zero over $\mathcal{L}$, so the sequence $\nabla\left(\cos \left(2 h_{k}\right), \sin \left(2 h_{k}\right)\right)$ is transverse to zero over $\mathcal{L}$. By Lemma 2.9 , this implies that $\nabla\left(\sigma_{k}^{2} / \sigma_{k}^{1}\right)$ is transverse to zero over a tubular neighborhood of $\mathcal{L}$ of fixed $g_{k}$-radius.

The transversality properties of Proposition 2.11 are $C^{2}$-open (see [3] or Definition 6.2 below), so we can follow Donaldson's ideas [10] and perturb the two sequences $\sigma_{k}^{1}, \sigma_{k}^{2}$ in order to achieve transversality over all of $M$. However, the condition of $\mathcal{L}$ projecting to a curve is neither generic nor open (generically it projects to an open set of $\mathbb{C P}^{1}$ ), so we cannot choose arbitrary Donaldson perturbations. Hence some work has to be done. The method that we follow is to perturb the quotient

$$
\frac{\sigma_{k}^{2}}{\sigma_{k}^{1}}=\frac{s_{k}^{1}+i s_{k}^{2}}{s_{k}^{1}-i s_{k}^{2}}
$$

using only real perturbations of the sections $s_{k}^{1}$ and $s_{k}^{2}$. Then the requirement that the sections be real forces the image of the Lagrangian under the perturbed map to remain in the unit circle.

An important remark to make at this point is that the property of being a Lefschetz pencil is invariant under projective automorphisms of $\mathbb{C P}^{1}$, and hence that the pair of sections $\left(s_{k}^{1}-i s_{k}^{2}, s_{k}^{1}+i s_{k}^{2}\right)$ defines a symplectic Lefschetz pencil if and only if the pair of real sections $\left(s_{k}^{1}, s_{k}^{2}\right)$ defines a symplectic Lefschetz pencil. Therefore, we only need to show how to perturb $s_{k}^{1}$ and $s_{k}^{2}$ among real sections in order to ensure that they define a Lefschetz pencil.

We proceed as follows. Symplectically identify a neighboorhood $U$ of $\mathcal{L}$ with a neighborhood $V$ of the zero section in $T^{*} \mathcal{L}$. We will define a number of structures over $T^{*} \mathcal{L}$, understanding that they translate to $U$ under this identification. Let $e: T^{*} \mathcal{L} \rightarrow T^{*} \mathcal{L}$ be the involution of $T^{*} \mathcal{L}$ defined by fiberwise multiplication by -1 and denoted by $\lambda$ the canonical Liouville form on $T^{*} \mathcal{L}$. We have $e^{*} \lambda=-\lambda$, and therefore $e^{*} d \lambda=-d \lambda$. There is a special class of compatible almost complex structures on $T^{*} \mathcal{L}$ defined by

$$
\mathcal{J}(\mathcal{L})=\left\{J \in \mathcal{J}\left(T^{*} \mathcal{L}, d \lambda\right): e^{*} J=-J\right\} .
$$

It is simple to check that the set of all these almost complex structures is contractible and non-empty. Let us call them symmetric almost complex structures. Construct $g(u, v)=d \lambda(u, J v)$ : then $e^{*} g=g$.

So far we have constructed a picture with a $\mathbb{Z}_{2}$-symmetry in $T^{*} \mathcal{L}$. Let us lift it to the bundle $L$. Over $T^{*} \mathcal{L}$ we can find a trivialization $L^{\otimes k} \cong T^{*} \mathcal{L} \times \mathbb{C}$ in which the connection is given by $\nabla=d+i A_{k}^{0}-i k \lambda$, where $d+i A_{k}^{0}$ is a flat connection such that $e^{*}\left(A_{k}^{0}\right)=A_{k}^{0}$ and $\left|A_{k}^{0}\right|_{g_{k}}=O\left(k^{-1 / 2}\right)$ everywhere. 
We can moreover assume that the section $\sigma_{\mathcal{L}, k}$ of $L^{\otimes k}$ over $\mathcal{L}$ corresponds to the constant function 1 in this trivialization. Using this trivialization, the involution $e$ lifts to an anti-complex isomorphism of line bundles

$$
\begin{aligned}
\hat{e}: \quad L^{\otimes k} & \longrightarrow L^{\otimes k} \\
(x, z) & \longmapsto(e(x), \bar{z}) .
\end{aligned}
$$

The connection induced by $\hat{e}$ on $L^{\otimes k}$ is $d-i A_{k}^{0}-i k \lambda$, which differs from $\nabla$ by $O\left(k^{-1 / 2}\right)$. Therefore, $\hat{e}^{*}(\bar{\partial})=\bar{\partial}+O\left(k^{-1 / 2}\right)$. We say that a complex bundle $E$ is symmetric if $e$ admits a lift to $E$ preserving its connection and complex structure. It is simple to check that the tangent bundle and any bundle obtained from it by tensor operations are symmetric bundles.

From all the previous remarks we get

Lemma 5.1. Let $E$ be a symmetric bundle. Let $s_{k}$ be an asymptotically holomorphic sequence of sections of the bundles $E \otimes L^{\otimes k}$ with respect to a symmetric almost complex structure. Then $\hat{e}^{*}\left(s_{k}\right)$ is also asymptotically holomorphic.

Moreover, if $s_{k}$ is $\epsilon$-transverse to zero, then $\hat{e}^{*}\left(s_{k}\right)$ is also $\epsilon$-transverse.

Definition 5.2. A section $s_{k}$ is called symmetric if $\hat{e}^{*}\left(s_{k}\right)=s_{k}$ in $U$ (and no restriction outside $U$ ).

Because we defined $\hat{e}$ using a trivialization of $L^{\otimes k}$ over $\mathcal{L}$ such that $\sigma_{\mathcal{L}, k}=$ 1 , we have

Lemma 5.3. A symmetric section of $L^{\otimes k}$ is real.

Moreover, just by checking the construction in the proof of Lemma 2.6 we can show

Lemma 5.4. The reference sections constructed in Lemma 2.6 can be chosen to be symmetric whenever $x \in \mathcal{L}$ if we fix a symmetric almost complex structure. Moreover, the asymptotically holomorphic sections constructed in Proposition 4.2 can also be chosen symmetric.

Proof. The reference sections can easily be chosen to be symmetric. Although a careful construction directly leads to symmetric sections when $x \in \mathcal{L}$, the most straightforward way is just to choose reference sections

$$
\hat{\sigma}_{k, x}^{\mathrm{ref}}=\frac{\sigma_{k, x}^{\mathrm{ref}}+\hat{e}^{*}\left(\sigma_{k, x}^{\mathrm{ref}}\right)}{2},
$$

that still satisfy all the properties of the Lemma. In fact, we can also use this method when $x \notin \mathcal{L}$, at the expense of obtaining sections that are concentrated not only near $x$ but rather near the two points $x$ and $e(x)$ (i.e., these sections satisfy suitably modified versions of the second and third statements in Lemma 2.6). 
The sections $s_{k}^{1}$ and $s_{k}^{2}$ constructed in Proposition 4.2 are also symmetric. To check this, one just has to check that the local sections $w_{j} \cos \left(P_{2, j}\right) \sigma_{k, x_{j}}^{\text {ref }}$ are symmetric. To make sure that this condition is fulfilled, we just replace that section by

$$
\frac{w_{j} \cos \left(P_{2, j}\right) \sigma_{k, x_{j}}^{\mathrm{ref}}+\hat{e}^{*}\left(w_{j} \cos \left(P_{2, j}\right) \sigma_{k, x_{j}}^{\mathrm{ref}}\right)}{2}
$$

which is a section that coincides with the previous one over $\mathcal{L}$ and satisfies the same properties (once again, since $x_{j} \in \mathcal{L}$ and since $P_{2, j}$ are real polynomials, with a careful construction this trick becomes unnecessary).

From now on we make the following assumptions:

(i) The almost complex structure $J$ is an extension to $M$ of a symmetric almost-complex structure in $U \cong V \subset T^{*} \mathcal{L}$.

(ii) $J$ is integrable and standard in Darboux coordinates over neighborhoods $B_{g_{k}}\left(p, c_{0} k^{1 / 2}\right)$ of the critical points of $f$ (this assumption was already made in Section 3).

(iii) $\sigma_{\mathcal{L}, k}$ is covariantly constant over a subset of $\mathcal{L}$ containing $B_{g_{k}}\left(p, c_{0} k^{1 / 2}\right)$ for every critical point of $f$ (see the remark after the statement of Lemma 2.2).

Moreover, as in Lemma 5.4, we assume that we are dealing with symmetric sections. In this context, Remark 2.7 still applies to our new symmetric reference sections near the critical points of $f$, since under the assumption that $\sigma_{\mathcal{L}, k}$ is locally parallel the involution $\hat{e}$ is connection-preserving, so that averaging with $\hat{e}^{*}\left(\sigma_{k, x}^{\text {ref }}\right)$ does not affect holomorphicity.

In Section 6, we will prove the following result:

Proposition 5.5. Fix $\delta>0$ small enough. There exists an asymptotically holomorphic sequence of symmetric sections $\tau_{k}^{1} \oplus \tau_{k}^{2}$ such that $\left|\tau_{k}^{1} \oplus \tau_{k}^{2}\right|_{C^{2}} \leq \delta$ and satisfying

(i) $s_{k}^{1}+\tau_{k}^{1}$ is $\epsilon$-transverse to zero over $M$, for some uniform $\epsilon>0$ (independent of $k$ ).

(ii) $\left(s_{k}^{1}+\tau_{k}^{1}\right) \oplus\left(s_{k}^{2}+\tau_{k}^{2}\right)$ is $\epsilon$-transverse to zero over $M$.

(iii) $\partial\left(\left(s_{k}^{2}+\tau_{k}^{2}\right) /\left(s_{k}^{1}+\tau_{k}^{1}\right)\right)$ is $\epsilon$-transverse to zero over the set $Z_{k, \epsilon}=$ $\left\{p \in M:\left|s_{k}^{1}+\tau_{k}^{1}\right| \geq \epsilon\right\}$.

Applying this result to our sequence we get a new sequence that we still denote by $s_{k}^{1} \oplus s_{k}^{2}$. The quotient of these two sections $s_{k}^{1}$ and $s_{k}^{2}$ or equivalently the quotient

$$
\frac{\sigma_{k}^{2}}{\sigma_{k}^{1}}=\frac{s_{k}^{1}+i s_{k}^{2}}{s_{k}^{1}-i s_{k}^{2}}
$$

satisfies the transversality estimates required of a symplectic Lefschetz pencil. Moreover, because the perturbation $\tau_{k}^{1} \oplus \tau_{k}^{2}$ can be chosen symmetric and 
$C^{2}$-small, the restriction $\left(\sigma_{k}^{2} / \sigma_{k}^{1}\right)_{\mid \mathcal{L}}$ remains a (circle-valued) Morse function. Now we want to perturb the sections $\sigma_{k}^{1}, \sigma_{k}^{2}$ in order to make the image of $\left.\left(\sigma_{k}^{2} / \sigma_{k}^{1}\right)\right|_{\mathcal{L}}$ an embedded curve and obtain a Morse function isotopic to the initial $h_{k}$.

Let $F_{k}=\left.\left(\sigma_{k}^{2} / \sigma_{k}^{1}\right)\right|_{\mathcal{L}}: \mathcal{L} \rightarrow S^{1}$. Then $F_{k}$ is $C^{2}$-close to $\left(\cos \left(2 h_{k}\right), \sin \left(2 h_{k}\right)\right)$, therefore it can be written in the form $\left(\cos \left(2 \hat{h}_{k}\right), \sin \left(2 \hat{h}_{k}\right)\right)$, where the function $\hat{h}_{k}$ is $C^{2}$-close to $h_{k}$. Extend $\hat{h}_{k}$ to the whole of $M$ keeping that $\left|\hat{h}_{k}\right|_{C^{2}, g_{k}}=O\left(k^{1 / 2}\right)$. Then define the pencil

$$
\tilde{F}_{k}=\frac{\sigma_{k}^{2} \cdot e^{\hat{h}_{k} / k}}{\sigma_{k}^{1}}
$$

This is still asymptotically holomorphic since it is a perturbation by $O\left(k^{-1 / 2}\right)$ of the previous map $F_{k}$, and it still satisfies the required transversality conditions. Moreover, the restriction of $\tilde{F}_{k}$ to $\mathcal{L}$ is equal to

$$
\exp \left(2 i \hat{h}_{k}+\frac{\hat{h}_{k}}{k}\right),
$$

which is isotopic to $\exp \left(2 i h_{k}+h_{k} / k\right)$, i.e., the composition of the initial $h_{k}$ with the embedding $\gamma(t)=\exp (2 i t+t / k)$ of $\left[0, k^{1 / 2}\right]$ into $\mathbb{C}$. Also, observe that these successive $C^{2}$-small perturbations affect in a controlled manner the critical points of $\tilde{F}_{k}$ along $\mathcal{L}$, which remain within arbitrarily small $g_{k^{-}}$ distance of those of $h_{k}$.

We now just follow Donaldson's proof in [10] to show that $\tilde{F}_{k}$ defines a symplectic Lefschetz pencil. The proof has three parts:

- We have to get the three transversality properties appearing in the statement of Proposition 2.11, either directly for $\tilde{F}_{k}$ or after composition with a projective automorphism of $\mathbb{C P}^{1}$.

- We have to check the local models near the base point set $\{p \in$ $\left.M: \sigma_{k}^{1}(p) \oplus \sigma_{k}^{2}(p)=0\right\}$.

- We have to check the local models close to the critical points of the map. By Donaldson's argument it automatically follows that the fibers are symplectic.

Proposition 5.5 covers the first part. The second one follows Donaldson's argument without any adaptation. The third one requires some work. We need the following auxiliary lemma:

Lemma 5.6. Let $p \in \mathcal{L}$ be a critical point of $f$ (or equivalently, of $h_{k}$ ). Then we can arrange that $\sigma_{k}^{2} / \sigma_{k}^{1}$ is J-holomorphic over $B_{g_{k}}(p, c)$ for some fixed constant $c>0$, with a non-degenerate critical point on $\mathcal{L}$ within distance $c / 2$ of $p$.

Proof. Let $x \in B_{g_{k}}\left(p, c_{0} k^{1 / 2}\right)$. If $d_{k}(p, x)<k^{1 / 6}-c$, then Remark 2.7 and the assumptions made above imply that $\sigma_{k, x}^{\text {ref }}$ is holomorphic over $B_{g_{k}}(p, c)$. 
If $d_{k}(p, x)>2 k^{1 / 6}+c$ then the reference section $\sigma_{k, x}^{\text {ref }}$ vanishes over $B_{g_{k}}(p, c)$. Finally, when $k^{1 / 6}-c<d_{k}(p, x)<2 k^{1 / 6}+c$, we can modify slightly the construction of the reference sections in equation (2.1), using a cut-off function which equals 1 inside a ball of $g_{k}$-radius $\frac{1}{2} k^{1 / 6}$ and 0 outside of a ball of $g_{k}$-radius $k^{1 / 6}-2 c$ (instead of the usual distances $k^{1 / 6}$ and $2 k^{1 / 6}$ ); this again ensures the vanishing of $\sigma_{k, x}^{\text {ref }}$ over $B_{g_{k}}(p, c)$. If we use these reference sections (and linear combinations of them with polynomial coefficients) throughout the construction (in particular in Proposition 5.5), then $\sigma_{k}^{1}$ and $\sigma_{k}^{2}$ are automatically holomorphic over $B_{g_{k}}(p, c)$.

Recall that by adjusting the parameter $\delta$ in Propositions 4.2 and 5.5, we can assume that $\left(\sigma_{k}^{2} / \sigma_{k}^{1}\right)_{\mid \mathcal{L}}$ is arbitrarily $C^{2}$-close to $\left(\cos \left(2 h_{k}\right), \sin \left(2 h_{k}\right)\right)$. In particular, because we have a uniform estimate on the Hessian of $h_{k}$ at its critical points, by choosing $\delta$ small enough in these two propositions we can ensure that the critical points of $\left(\sigma_{k}^{2} / \sigma_{k}^{1}\right)_{\mid \mathcal{L}}$ are non-degenerate and lie within distance $c / 2$ of those of $h_{k}$.

With the above understood, if $p$ is a critical point of $h_{k}$ then $\sigma_{k}^{2} / \sigma_{k}^{1}=$ $F_{k}(z)$ is holomorphic around $p$. So, when we perturb $F_{k}$ in order to make the image of $\mathcal{L}$ an embedded curve, we can choose the function $\hat{h}_{k}$ in equation (5.1) (with controlled $C^{2}$-norm, taking real values over $\mathcal{L}$, and such that $F_{k}=\left(\cos \left(2 \hat{h}_{k}\right), \sin \left(2 \hat{h}_{k}\right)\right)$ over $\left.\mathcal{L}\right)$ to be holomorphic in a neighborhood of uniform $g_{k}$-radius of every critical point. This means that throughout our construction we have preserved holomorphicity near the critical points which lie on $\mathcal{L}$.

The last part of Donaldson's argument [10] involves a perturbation of the pencil map near its critical points in order to achieve the correct (holomorphic quadratic) local model. However, we have already ensured holomorphicity near the critical points in $\mathcal{L}$ (any critical point of $\tilde{F}_{k}$ along $\mathcal{L}$ is also a critical point of $\hat{h}_{k}$ and hence lies close to a critical point of $f$ ). Hence, this perturbation is only needed at those critical points which lie outside of $\mathcal{L}$. Because $\nabla h_{k}$ is $\eta$-transverse to zero over $\mathcal{L}$ we can find a uniform lower bound on the $g_{k}$-distance between such a critical point and $\mathcal{L}$. Therefore the required perturbation can be carried out without affecting the behavior of $\tilde{F}_{k}$ along $\mathcal{L}$, thus completing the proof of Theorem 1.3.

\section{Globalization preserving symmetry: proof of Proposition 5.5.}

6.1. Proof of the first part of Proposition 5.5. We have to follow Donaldson's ideas in [9]. Let us review how to make a section $s_{k}$ transverse to zero. The steps to follow are

(i) Given a point $x \in M$, we use the chart $\Psi_{k, x}$ provided by Lemma 2.3 to trivialize in an approximately holomorphic way a neighborhood of fixed $g_{k}$-radius of $x$. We also trivialize the bundle $L^{\otimes k}$ in that neighborhood 
using the section $\sigma_{k, x}^{\text {ref }}$ given by Lemma 2.6. This allows us to construct an asymptotically holomorphic function

$$
\begin{aligned}
f_{k, x}: B(0,1) \subset \mathbb{C}^{n} & \longrightarrow \mathbb{C} \\
y & \longmapsto \frac{s_{k}\left(\Psi_{k, x}^{-1}(y)\right)}{\sigma_{k, x}^{\mathrm{ref}}\left(\Psi_{k, x}^{-1}(y)\right)} .
\end{aligned}
$$

(ii) We perturb the function to a new one which is transverse to zero by adding a small constant $w$ (this amounts to adding $w \sigma_{k, x}^{\text {ref }}$ to $s_{k}$ ). The precise result is

Lemma 6.1 (Donaldson). There exists an integer $p$ depending only on the dimension $n$, with the following property: let $\delta$ be a constant with $0<\delta<1 / 2$ and $\sigma=\delta\left(\log \left(\delta^{-1}\right)\right)^{-p}$. Let $f: B(0,11 / 10) \subset \mathbb{C}^{n} \rightarrow \mathbb{C}$ satisfy

$$
|f| \leq 1, \quad|\bar{\partial} f| \leq \sigma, \quad|\nabla \bar{\partial} f| \leq \sigma .
$$

Then there exists a complex number $w$ with $|w| \leq \delta$, such that $f-w$ is $\sigma$-transverse to zero in the unit ball of $\mathbb{C}^{n}$.

(iii) The third part of the argument consists of a method to achieve transversality in the entire manifold from the local transversality argument. We start by fixing a covering of the manifold by a finite number of Darboux charts $\left\{U_{i}\right\}_{i=1}^{N}$. It is enough to know how to get transversality in one of them. For we can start by perturbing $s_{k}$ to some $s_{k}^{1}=s_{k}+\sigma_{k}^{1}$ which is $\epsilon_{1}$-transverse to zero in $U_{1}$ and then we perturb every $s_{k}^{i}$ to $s_{k}^{i+1}=s_{k}^{i}+\sigma_{k}^{i+1}$ which is $\eta_{i}$-transverse to zero in $U_{i+1}$. If $s_{k}^{i}$ is $\epsilon_{i}$-transverse to zero in $\cup_{j=1}^{i} U_{j}$ and the perturbation is small enough then $s_{k}^{i+1}$ is still $\epsilon_{i} / 2$-transverse to zero in $\cup_{j=1}^{i} U_{j}$. This is because transversality is a $C^{1}$-open property, in the following sense:

Definition 6.2. A property $\mathcal{P}(\epsilon, x)$ of a sequence of sections $s_{k}: M \rightarrow$ $E_{k}$ is $C^{r}$-open if there exists a uniform constant $c_{0}>0$ such that if $s_{k}$ has the property $\mathcal{P}(\epsilon, x)$ then any $s_{k}^{\prime}$ such that $\left|s_{k}(x)-s_{k}^{\prime}(x)\right|_{C^{r}} \leq c_{0} \epsilon$ has the property $\mathcal{P}(\epsilon / 2, x)$.

Choosing $\epsilon_{i+1}=\min \left\{\epsilon_{i} / 2, \eta_{i}\right\}, s_{k}^{i+1}$ is $\epsilon_{i+1}$-transverse to zero in $\cup_{j=1}^{i+1} U_{j}$.

The difficult part is to get transversality in a single Darboux chart. Take the lattice $(c \mathbb{Z})^{2 n} \subset \mathbb{R}^{2 n}$, for some small $c>0$. The image of the lattice in $M$ will be denoted by $\Gamma$. We perturb our section $s_{k}$ with sections centered at the different points of the lattice $\Gamma$. An argument of Donaldson [9] shows a way of doing this which yields uniform transversality over the entire Darboux chart, with a transversality amount independent of $k$, in spite of the increasing number of lattice points needed to cover the rescaled Darboux chart. The crucial 
ingredients here are: (a) transversality is an open property and (b) the sections $\sigma_{k, x}^{\text {ref }}$ decay exponentially away from $x$. These two features make it possible to keep track of the mutual interference created by different perturbations. We do not provide the details, which can be found in $[\mathbf{9}]$.

In our case, we have to adapt the argument to perturb an asymptotically holomorphic sequence of symmetric sections $s_{k}^{1}$ to make it transverse to zero. This means that we want the construction to be invariant by the involution $\hat{e}$.

As above, we start by covering the manifold by a finite number of Darboux balls $U_{j}$, chosen in such a way that the Darboux balls which intersect the Lagrangian submanifold $\mathcal{L}$ satisfy $e\left(U_{j}\right)=U_{j}$. As before, we need to get transversality for a single chart. If $U_{j}$ does not intersect $\mathcal{L}$ then we use Donaldson's argument without modification. If the chart intersects $\mathcal{L}$ then we have to be more careful. We will get transversality in pairs of small balls $V$ and $e(V)$ at the same time. Let us review the adaptations needed in each of the three parts of the argument:

(i) For the first part, there is no change. We trivialize our section $s_{k}^{1}$ in a neighborhood of a point $x$, using the trivializing section $\sigma_{k, x}^{\text {ref }}$ to obtain a complex-valued function $f$ defined by

$$
f=\frac{s_{k}^{1}}{\sigma_{k, x}^{\mathrm{ref}}} .
$$

(ii) We also follow the structure of the second part. Now, the section $w \sigma_{k, x}^{\mathrm{ref}}+\bar{w} \hat{e}^{*} \sigma_{k, x}^{\mathrm{ref}}$ is symmetric for any complex number $w$ and can therefore be used to locally perturb $s_{k}^{1}$. After division by $\sigma_{k, x}^{\text {ref }}$, replacing $s_{k}^{1}$ by $s_{k}^{1}-w \sigma_{k, x}^{\text {ref }}-\bar{w} \hat{e}^{*} \sigma_{k, x}^{\text {ref }}$ corresponds to a perturbation of $f$ to the new function

$$
f_{(w)}=f-w-\bar{w} \frac{\hat{e}^{*} \sigma_{k, x}^{\mathrm{ref}}}{\sigma_{k, x}^{\mathrm{ref}}},
$$

Now we apply the following

Proposition 6.3. Let $C>0, \kappa>0$. There exists an integer $p$ and a fixed $\delta_{0}>0$ (depending only on $C, \kappa$ and the dimension) verifying the following property: for $0<\delta<\delta_{0}$, let $\sigma=\delta\left(\log \left(\delta^{-1}\right)\right)^{-p}$. Let $f$, $h$ be two complex-valued functions defined over the ball $B^{+}=B\left(0, \frac{11}{10}\right) \subset \mathbb{C}^{n}$ satisfying the following bounds over $B^{+}$,

$$
\begin{gathered}
|f| \leq 1, \quad|\bar{\partial} f| \leq \sigma, \quad|\nabla \bar{\partial} f| \leq \sigma \\
|h| \leq 1-\kappa, \quad|d h| \leq C, \quad|\bar{\partial} h| \leq \sigma, \quad|\nabla \bar{\partial} h| \leq \sigma .
\end{gathered}
$$

Then there exists $w \in \mathbb{C}$ with $|w|<\delta$ such that $f-w-\bar{w} h$ is $\sigma$ transverse to 0 over the unit ball in $\mathbb{C}^{n}$. The same result holds for 
1-parameter families of functions $f_{t}, h_{t}$ depending continuously on $t \in$ $[0,1]$, where we obtain a continuous path $w_{t} \in \mathbb{C}$, with $\left|w_{t}\right|<\delta$.

The proof of this Proposition will be given in Section 7; it is essentially a careful modification of Donaldson's local Lemma.

Recall from the beginning of Section 5 that the desired transversality property already holds over a neighborhood of $\mathcal{L}$ of uniform $g_{k}$-radius, say $\rho>0$, and will automatically be preserved by all perturbations of $s_{k}^{1}$ that are sufficiently $C^{1}$-small. Therefore, in the construction, we only need to consider the case where $x$ is at distance at least $\rho$ from $\mathcal{L}$, and rescaling the coordinates by a constant factor if necessary we can assume that the ball $B^{+}$does not intersect the $(\rho / 2)$-neighborhood of $\mathcal{L}$. This implies that $h=\hat{e}^{*}\left(\sigma_{k, x}^{\mathrm{ref}}\right) / \sigma_{k, x}^{\mathrm{ref}}$ satisfies a bound of the form $|h| \leq 1-\kappa$ over $B^{+}$for a uniform constant $\kappa>0$, thus allowing us to apply Proposition 6.3.

Then the new section $s_{k}^{1}-w \sigma_{k, x}^{\mathrm{ref}}-\bar{w} \hat{e}^{*}\left(\sigma_{k, x}^{\mathrm{ref}}\right)$ is $\sigma$-transverse to zero in a ball $B_{x}$ of fixed radius around $x$. Moreover, it is symmetric as desired, and therefore by symmetry it is also $\sigma$-transverse to zero over $e\left(B_{x}\right)$.

(iii) The third part remains unchanged except for the fact that we choose the lattice $\Gamma$ to be $e$-invariant, and the transversality is obtained simultaneously over pairs of balls centered at points $x, e(x)$, for all $x \in \Gamma$ which lie at $g_{k}$-distance at least $\rho$ from $\mathcal{L}$ (while transversality already holds over the $\rho$-neighborhood of $\mathcal{L}$ by assumption).

6.2. Proof of the second part of Proposition 5.5. Now we have to get transversality for the sequence $s_{k}^{1} \oplus s_{k}^{2}$ while preserving $e$-invariance. Instead of using the generalization of the local transversality Lemma 6.1 to maps from $\mathbb{C}^{n}$ to $\mathbb{C}^{r}$ with $r>1$ obtained by Donaldson in [10], we shall adapt the ideas of $[\mathbf{2}]$ to our $e$-invariant setting. This is because we have not developed the extension of Proposition 6.3 for maps from $\mathbb{C}^{n}$ to $\mathbb{C}^{r}$. Such a result should hold, but it is easier for the purposes of this article just to carefully use the case $r=1$.

Because of the transversality of the sequence $s_{k}^{1}$ achieved by means of a symmetric perturbation in Subsection 6.1 , the zero set $Z\left(s_{k}^{1}\right)$ of the section $s_{k}^{1}$ is a symplectic submanifold for $k$ large enough, and the tangent spaces to $Z\left(s_{k}^{1}\right)$ are within $O\left(k^{-1 / 2}\right)$ of being complex subspaces of $(T M, J)$. We perturb $s_{k}^{2}$ in order to make its restriction to the submanifold $Z\left(s_{k}^{1}\right)$ transverse to zero. The argument is similar to the one used to get transversality of $s_{k}^{1}$ all over $M$, however, some details concerning the fact that the submanifold $Z\left(s_{k}^{1}\right)$ changes with $k$ have to be taken into account. We refer the reader to [2] for more details. In our case, the only required adaptation is to impose symmetry for all the perturbations. However, this works exactly in the same way as in Subsection 6.1 and only requires us to use Proposition 6.3. 
Once we have perturbed the sequence $s_{k}^{2}$ into one that is $\epsilon$-transverse to zero on $Z\left(s_{k}^{1}\right)$, we conclude with the following linear algebra lemma:

Lemma 6.4 (Subsection 3.6 in [2]). Let $s_{k}^{1} \oplus s_{k}^{2}$ be an asymptotically holomorphic sequence of sections of the bundles $E_{k}=\left(E_{1} \oplus \mathbb{C}\right) \otimes L^{\otimes k}$. Assume that $s_{k}^{1}$ is $\epsilon$-transverse to zero on an open set $U$. Assume that $s_{k}^{2}$ is $\epsilon$ transverse to zero on $Z\left(s_{k}^{1}\right) \cap U$. Then $s_{k}^{1} \oplus s_{k}^{2}$ is $\epsilon^{\prime}$-transverse to zero over $U$, where $\epsilon^{\prime}>0$ depends only on $\epsilon$ and the uniform bound on $\left|\nabla \nabla\left(s_{k}^{1} \oplus s_{k}^{2}\right)\right|$, but not on $k$.

6.3. Proof of the third part of Proposition 5.5. From the previous steps of the argument, we may assume that there exists an uniform $\epsilon>0$ for which

- $s_{k}^{1} \oplus s_{k}^{2}$ is $\epsilon$-transverse to zero over $M$.

- $s_{k}^{1}$ is $\epsilon$-transverse to zero over $M$.

- $s_{k}^{2}$ is $\epsilon$-transverse to zero over $M$.

So, our only remaining task is to modify $s_{k}^{1} \oplus s_{k}^{2}$ by symmetric perturbations in order to achieve transversality of the sequence $\partial\left(s_{k}^{2} / s_{k}^{1}\right)$ over the set $Z_{k, \epsilon}$. As in Subsection 6.1, the $C^{2}$-openness of this condition (see Definition 6.2) makes it possible to reduce the problem to a fixed Darboux chart $U$; if $U$ does not intersect the Lagrangian submanifold $\mathcal{L}$, then we just refer to the standard proof in [10]. In the other case, let $U$ be a Darboux chart intersecting $\mathcal{L}$, such that $e(U)=U$, and fix a set of sections $\left\{\alpha_{j}\right\}_{j=1}^{n}$ of the topologically trivial bundle $\left(T^{*} U\right)^{1,0}$, defining an orthonormal basis at every point of $U$. Note that we do not impose any integrability condition on the sections. Define the line subbundles $A_{r}=\mathbb{C} \alpha_{r} \subset\left(T^{*} U\right)^{1,0}$, and the corresponding filtration $E_{j}=\bigoplus_{r=1}^{j} A_{r}$. The decomposition $\left(T^{*} U\right)^{1,0}=$ $E_{n}=\bigoplus_{r=1}^{n} A_{r}$ allows us to define projection operators $\pi_{r}: E_{n} \rightarrow A_{r}$ and $\pi_{\leq r}: E_{n} \rightarrow E_{r}$. We consider the section $\left.\partial\left(s_{k}^{2} / s_{k}^{1}\right)\right|_{U}: U \rightarrow E_{n}$, and introduce the following definition:

Definition 6.5. A section $s_{k}^{1} \oplus s_{k}^{2}$ of $\mathbb{C}^{2} \otimes L^{\otimes k}$ is $\left(\epsilon, E_{r}\right)$-sharp at a point $x \in U$ if the section $\pi_{\leq r}\left(\partial\left(s_{k}^{2} / s_{k}^{1}\right)\right)$ of $E_{r}$ is $\epsilon$-transverse to zero at $x$.

Note that this property is $C^{2}$-open as defined in Definition 6.2. This allows us to use the three step process described in Subsection 6.1 to achieve sharpness at every point of $U \cap Z_{k, \epsilon}$. Moreover, $E_{n}$-sharpness is equivalent to transversality to zero for $\partial\left(s_{k}^{2} / s_{k}^{1}\right)$ and $E_{0}$-sharpness is a void condition.

The scheme of the proof is to assume $\left(\epsilon, E_{r}\right)$-sharpness and then manage to achieve $\left(\epsilon^{\prime}, E_{r+1}\right)$-sharpness. Denote by $W_{k, r}$ the zero set of $\pi_{\leq r}\left(\partial\left(s_{k}^{2} / s_{k}^{1}\right)\right)$, which is a symplectic submanifold for $k$ large enough. We proceed by making the following definition: 
Definition 6.6. A section $s_{k}^{1} \oplus s_{k}^{2}$ is $\left(\epsilon, A_{r+1}\right)$-sharp at a point $x \in U \cap W_{k, r}$ if the restriction to $W_{k, r}$ of the section $\pi_{r+1}\left(\partial\left(s_{k}^{2} / s_{k}^{1}\right)\right)$ of $A_{r+1}$ is $\epsilon$-transverse to zero at $x$.

This is again a $C^{2}$-open property under the assumption of $E_{r}$-sharpness (because $W_{k, r}$ then depends continuously on $s_{k}^{1} \oplus s_{k}^{2}$ ). By Lemma 6.4, we know that $E_{r+1}$-sharpness on $U$ follows from $A_{r+1}$-sharpness on $W_{k, r} \cap U$.

We analyze the three steps needed for the argument:

(i) Fix a point $x \in W_{k, r}$. We use Lemma 2.3 to define a Darboux chart $\Psi_{k, x}$ centered at $x$. After applying a linear transformation in $U(n)$, we can ensure that, at the point $x$,

$$
\alpha_{j}(x)=\left(\Psi_{k, x}^{*}\left(\partial z_{j}\right)\right)(x) .
$$

Also, recall from the beginning of Section 5 that we can assume $x$ lies at a $g_{k}$-distance bigger than a uniform constant from $\mathcal{L}$ (indeed, the desired tranversality property over a small tubular neighborhood of $\mathcal{L}$ follows from the estimates achieved in Section 4). This implies the existence of a uniform lower bound on at least one of the coordinates at $e(x)$, say $z_{j_{0}}$. Let $\gamma_{x}=\Psi_{k, x}^{*}\left(z_{j_{0}}\right)(e(x))$, and for $w \in \mathbb{C}$ define

$$
s_{k ;(w)}^{2}=s_{k}^{2}+w \Psi_{k, x}^{*}\left(z_{r+1}\left(1-\gamma_{x}^{-1} z_{j_{0}}\right)^{2}\right) \sigma_{k, x}^{\mathrm{ref}} .
$$

Since we have a uniform upper bound on $\gamma_{x}^{-1}$, the perturbations we add to $s_{k}^{2}$ are asymptotically holomorphic and have uniform exponential decay away from $x$, as required for the general construction. For small enough values of $w$ the submanifold $W_{k, r ;(w)}=\left\{\pi_{\leq r}\left(\partial\left(s_{k ;(w)}^{2} / s_{k}^{1}\right)\right)=0\right\}$ is a small deformation of $W_{k, r}$ and can be viewed as the image by the exponential map of the graph of a section of the normal bundle to $W_{k, r}$. With this understood, we can define

$$
\zeta_{k, x}=\left.\frac{\partial}{\partial w}\right|_{w=0}\left(\left.\pi_{r+1}\left(\partial\left(\frac{s_{k ;(w)}^{2}}{s_{k}^{1}}\right)\right)\right|_{W_{k, r ;(w)}}\right) .
$$

Observe that $\zeta_{k, x}(x)=\left(\alpha_{r+1}(x) \sigma_{k, x}^{\text {ref }}(x) / s_{k}^{1}(x)\right)$ is uniformly bounded from below, and so there is a uniform radius $\rho>0$ such that $\zeta_{k, x}$ is bounded from below by a uniform constant over $W_{k, r} \cap B_{g_{k}}(x, \rho)$. Moreover, by construction, the perturbation we add to $s_{k}^{2}$ vanishes up to order 2 at $e(x)$, so $\zeta_{k, x}(e(x))=0$. Hence, decreasing $\rho$ if necessary (but preserving uniformity) we can assume that a bound of the form $\left|\hat{e}^{*} \zeta_{k, x}\right| \leq(1-\kappa)\left|\zeta_{k, x}\right|$ holds at all points of $W_{k, r} \cap B_{g_{k}}(x, \rho)$.

Finally, recall from $[2]$ that, decreasing $\rho$ if necessary, we can trivialize the submanifold $W_{k, r}$ in the ball of radius $2 \rho$ around $x$. More precisely, using Lemma 4 of $[\mathbf{2}]$ we can get an approximately holomorphic chart $\Phi_{k, x}: B\left(0, \frac{11}{10}\right) \subset \mathbb{C}^{n-r} \rightarrow W_{k, r}$. Using these trivializations, 
we can introduce a function $f_{k, r+1}: B\left(0, \frac{11}{10}\right) \subset \mathbb{C}^{n-r} \rightarrow \mathbb{C}$, defined by

$$
f_{k, r+1}=\Phi_{k, x}^{*}\left(\frac{\pi_{r+1}\left(\partial\left(s_{k}^{2} / s_{k}^{1}\right)\right)}{\zeta_{k, x}}\right),
$$

describing the restriction to $W_{k, r}$ of the component of $\partial\left(s_{k}^{2} / s_{k}^{1}\right)$ along $\alpha_{r+1}$ and satisfying asymptotically holomorphic estimates.

(ii) If $x$ lies on $\mathcal{L}$ or within a small tubular neighborhood of uniform $g_{k^{-}}$ radius, then the transversality estimates obtained on $\mathcal{L}$ at the beginning of Section 5 imply the desired result, and no work is required. Otherwise, we apply Proposition 6.3 to get a constant $w$ such that

$$
f_{k, r+1 ;(w)}=f_{k, r+1}-w-\bar{w} \Phi_{k, x}^{*}\left(\frac{\hat{e}^{*}\left(\zeta_{k, x}\right)}{\zeta_{k, x}}\right)
$$

is $\sigma$-transverse to zero over the unit ball. Now we define

$$
\begin{aligned}
\sigma_{k}^{2}= & w \Psi_{k, x}^{*}\left(z_{r+1}\left(1-\gamma_{x}^{-1} z_{j_{0}}\right)^{2}\right) \sigma_{k, x}^{\mathrm{ref}} \\
& +\hat{e}^{*}\left(w \Psi_{k, x}^{*}\left(z_{r+1}\left(1-\gamma_{x}^{-1} z_{j_{0}}\right)^{2}\right) \sigma_{k, x}^{\mathrm{ref}}\right) .
\end{aligned}
$$

Clearly, the new sequence $s_{k}^{1} \oplus\left(s_{k}^{2}-\sigma_{k}^{2}\right)$ corresponds to the desired perturbation (i.e., replacing $f_{k, r+1}$ with $f_{k, r+1 ;(w)}$ in the trivialization), up to higher order terms (bounded by $O\left(w^{2}\right)$ ), which are negligible, i.e., much smaller than $\sigma$ (recall that $\sigma=\delta\left(\log \left(\delta^{-1}\right)\right)^{-p}$ is the constant appearing in Proposition 6.3). This gives us $\left(\sigma, A_{r+1}\right)$-sharpness over a ball of fixed $g_{k}$-radius in $W_{k, r}$. Also, by construction, the perturbation (6.1) is $\hat{e}$-invariant.

(iii) The final step (patching together the various local perturbations to achieve sharpness globally) is the same as before: we again choose the lattice $\Gamma$ to be $e$-invariant and in order to be able to apply Proposition 6.3 we discard those points of $\Gamma$ which lie inside the small neighborhood of $\mathcal{L}$ over which the initially given sections already satisfy all desired properties.

\section{The parametric case}

Theorem 1.4 is proved using the same strategy as Theorem 1.3, replacing all the objects in the above argument by families indexed by the parameter $t \in[0,1]$. The existence of families of Darboux charts $\Psi_{k, x, t}$ and reference sections $\sigma_{k, x, t}^{\text {ref }}$ that depend continuously on $t$, as well as 1-parameter versions of the various standard results in approximately holomorphic geometry, are well-known $[\mathbf{2}, \mathbf{1 0}]$. It is worth noting that since the isotopy $\left(\mathcal{L}_{t}\right)_{t \in[0,1]}$ is Hamiltonian and hence induced by a family of global symplectomorphisms $\psi_{t}$ of $M$, after pullback via $\psi_{t}$ we can assume that the Lagrangian submanifold is fixed (while all the other auxiliary data in the construction depend on $t$ ). Although the standard results can be used to achieve transversality along 
submanifolds that depend on $t$ (see, e.g., the arguments in [2]), keeping $\mathcal{L}$ fixed makes it easier to adapt the argument to the parametric case.

In any case, all that is required is to check that, at every stage of the construction of adapted pencils (throughout Sections 3-6), it is possible to interpolate between the choices of sections and perturbations which lead to the given pencils $\phi_{k, 0}, \phi_{k, 1}$ via 1-parameter families of objects that depend continuously on $t$. The two places in the argument where the adaptation to the parametric case is not completely straightforward are the existence of the real trivialization $\sigma_{\mathcal{L}, k}$ (Lemma 2.2), and the possibility of achieving estimated transversality via symmetric perturbations (Proposition 6.3).

The existence of a continuous family of sections $\sigma_{\mathcal{L}_{t}, k}$ of $L^{\otimes k}$ over $\mathcal{L}_{t}$ which satisfy the properties of Lemma 2.2 and extend the given choices for $t=0$ and $t=1$ is guaranteed by the requirement that $\pi_{1}\left(\mathcal{L}_{t}\right)=1$. This ensures that the non-vanishing sections $\sigma_{\mathcal{L}_{0}, k}$ and $\sigma_{\mathcal{L}_{1}, k}$ used to build the pencils $\phi_{k, 0}$ and $\phi_{k, 1}$ belong to the same homotopy class. Even if $\pi_{1}\left(\mathcal{L}_{t}\right) \neq 1$, Theorem 1.4 still holds when the isotopy $\left\{\mathcal{L}_{t}\right\}_{0 \leq t \leq 1}$ is generated by a family of global symplectomorphisms of $M$, provided that the linking numbers of the fibers of the pencil with all closed loops on the Lagrangian submanifold are assumed to be equal for $\phi_{k, 0}$ and $\phi_{k, 1}$ (or equivalently, assuming that $\sigma_{\mathcal{L}_{0}, k}$ and $\sigma_{\mathcal{L}_{1}, k}$ are homotopic). Note that, if the isotopy $\left\{\mathcal{L}_{t}\right\}$ is not generated by global symplectomorphisms, then Stokes' theorem precludes the existence of a continuous family of non-vanishing sections $\sigma_{\mathcal{L}_{t}, k}$ such that $\left|\nabla \sigma_{\mathcal{L}_{t}, k}\right|=O\left(k^{-1 / 2}\right)$.

Finally, concerning Proposition 6.3, we give below a proof that adapts easily to the parametric case (in the non-parametric case it would be easier to follow the argument in $[\mathbf{9}]$ and show that $w$ can be chosen real).

Proof of Proposition 6.3. As in [2, 9], we can approximate $f$ and $h$ by polynomials $p$ and $q$ such that

$$
\begin{aligned}
& |f-p|_{C^{1}} \leq c \sigma, \operatorname{deg}(p) \leq c \log \left(\sigma^{-1}\right), \\
& |h-q|_{C^{1}} \leq c \sigma, \operatorname{deg}(q) \leq c \log \left(\sigma^{-1}\right),
\end{aligned}
$$

in the unit ball, for $c>0$ a constant. Now define the function

$$
s(z, w)=p(z)-w-\bar{w} q(z) .
$$

For $\sigma$ small enough (choosing $\delta_{0}$ appropriately), we get that $|q| \leq 1-\frac{\kappa}{2}$. Therefore

$$
\frac{\partial s}{\partial w}=-\boldsymbol{I}-\frac{\partial \bar{w}}{\partial w} \cdot q
$$

is an invertible matrix (here $\partial s / \partial w$ means the Jacobian matrix of the function $s$ with respect to the two variables $\operatorname{Re}(w), \operatorname{Im}(w))$. Its inverse satisfies

$$
\left|\left(\frac{\partial s}{\partial w}\right)^{-1}\right| \leq 2 \kappa^{-1} \text {. }
$$


We can apply the implicit function theorem to $s(z, w)$ to obtain a (unique) function $w=w(z)$ such that $s(z, w(z))=0$. This function is defined over $B$ and, taking derivatives, we get

$$
\frac{\partial s}{\partial z}+\frac{\partial s}{\partial w} \frac{d w}{d z}=0
$$

So

$$
\frac{d w}{d z}=-\left(\frac{\partial s}{\partial w}\right)^{-1} \frac{\partial s}{\partial z}
$$

along the graph of $w=w(z)$. This implies that

$$
\left|\frac{d w}{d z}\right| \leq 2 \kappa^{-1}|l(z)|, \quad \text { where } l(z)=\frac{\partial s}{\partial z}(z, w(z)) .
$$

Recall that $\partial s / \partial z$ is the Jacobian matrix of the partial derivatives of $s$ with respect to $\operatorname{Re}(z)$ and $\operatorname{Im}(z)$, which we evaluate along the graph of $w=w(z)$. Now consider the set

$$
Y_{l, \sigma}=\{z \in B:|l(z)| \leq \sigma\} .
$$

Given constants $c_{1}, c_{2}>0$, denote by $N_{c_{2} \sigma}\left(w\left(Y_{l, c_{1} \sigma}\right)\right)$ the $c_{2} \sigma$-neighborhood of the image $w\left(Y_{l, c_{1} \sigma}\right) \subset \mathbb{C}$. Suppose that $w_{0}$ is a point outside of this neighborhood. Let us see that $s\left(z, w_{0}\right)$ is $c_{0} \sigma$-transverse to zero over $B$, for some constant $c_{0}>0$.

Take a point $z \in B$ with $\left|s\left(z, w_{0}\right)-s(z, w(z))\right|=\left|s\left(z, w_{0}\right)\right|<\frac{1}{2} \kappa c_{2} \sigma$. Then $\left|w(z)-w_{0}\right|<c_{2} \sigma$ by equation (7.2). Therefore $z$ is not in $Y_{l, c_{1} \sigma}$ and so $|l(z)| \geq c_{1} \sigma$. This is rewritten as

$$
\left|\frac{\partial s}{\partial z}(z, w(z))\right| \geq c_{1} \sigma .
$$

At this point we apply the bound

$$
\left|\frac{\partial^{2} s}{\partial z \partial w}\right|=\left|\frac{d q}{d z}\right| \leq C+1
$$

for $\sigma$ small enough. Decreasing $c_{2}$ if necessary we can assume that $c_{1}>$ $2(C+1) c_{2}$, and then

$$
\left|\frac{\partial s}{\partial z}\left(z, w_{0}\right)\right| \geq c_{1} \sigma-(C+1) c_{2} \sigma \geq \frac{c_{1}}{2} \sigma .
$$

Therefore $s\left(z, w_{0}\right)$ is $c_{0} \sigma$-transverse to zero with $c_{0}=\min \left\{\frac{1}{2} c_{1}, \frac{1}{2} \kappa c_{2}\right\}$.

Finally, recall that transversality is a $C^{1}$-open property, and that (assuming $\left.\left|w_{0}\right|<1\right)$ the bounds $(7.1)$ imply that $\left|s\left(z, w_{0}\right)-\left(f-w_{0}-\bar{w}_{0} h\right)\right|_{C^{1}} \leq 2 c \sigma$. Since $s\left(z, w_{0}\right)$ is $c_{0} \sigma$-transverse to zero, it follows that $f-w_{0}-\bar{w}_{0} h$ is $c_{0} \sigma / 2$ transverse to zero if the constant $c_{0}$ is large enough compared to $c$.

It remains to see that there are points in $B(0, \delta)-N_{C \sigma}\left(w\left(Y_{l, C \sigma}\right)\right)$ for $C$ a large constant. For this we use the following result (Proposition 25 of $[\mathbf{9}]$ ): 
Lemma 7.1. Let $F: \mathbb{R}^{m} \rightarrow \mathbb{R}$ be a polynomial function of degree $d$, and let $S(\theta)=\left\{x \in \mathbb{R}^{m}:|x| \leq 1, F(x) \leq 1+\theta\right\}$. Then for arbitrarily small $\theta>0$ there exist constants $C$ and $\nu$ depending only on the dimension $m$ such that $S(0)$ may be decomposed into pieces $S(0)=S_{1} \cup \cdots \cup S_{A}$, where $A \leq C d^{\nu}$, in such a way that any pair of points in the same piece $S_{r}$ can be joined by a path in $S(\theta)$ of length less than $C d^{\nu}$.

Now it is necessary to give a more explicit description of the function $w(z)$. Since it solves the equation $p(z)=w(z)+\bar{w}(z) q(z)$, we have

$$
w=\frac{p-\bar{p} q}{1-|q|^{2}}
$$

Therefore $w(z)\left(1-|q|^{2}\right)$ is a polynomial function of the $2 n$ real variables $\operatorname{Re}\left(z_{j}\right), \operatorname{Im}\left(z_{j}\right)$. Hence the condition $|l(z)| \leq C \sigma$ can be rewritten as $|l(z)|^{2}\left(1-|q|^{2}\right)^{2} \leq C^{2} \sigma^{2}\left(1-|q|^{2}\right)^{2}$, which is equivalent to

$$
\left|\left(1-|q|^{2}\right) \frac{d p}{d z}-\left(1-|q|^{2}\right) \bar{w}(z) \frac{d q}{d z}\right|^{2}-C^{2} \sigma^{2}\left(1-|q|^{2}\right)^{2}+1 \leq 1 .
$$

Let $F$ be the polynomial on the left hand side of equation (7.4), so that $S(0)$ is $Y_{l, C \sigma}$. By Lemma 7.1, there is a polynomial $P(d)$ such that $S(0)$ can be decomposed into at most $P(d)$ subsets, such that two points in the same subset can be joined by a path of length at most $P(d)$ inside $Y_{l, 2 C \sigma}$ (choose $\theta=3 C^{2} \kappa^{2} \sigma^{2} / 4 \leq 3 C^{2} \sigma^{2}\left(1-|q|^{2}\right)^{2}$, since $\left(1-|q|^{2}\right)^{2}>\kappa^{2} / 4$ ). Using the bound (7.3) on $|d w / d z|$, this implies that the image $N_{C \sigma}\left(w\left(Y_{l, C \sigma}\right)\right)$ is contained in the union of $P(d)$ discs of radius at most $\left(4 \kappa^{-1} P(d)+1\right) C \sigma$ (see the argument in [2, p. 976-977]). This proves that for some large fixed $p>0$, if $\sigma=\delta\left(\log \left(\delta^{-1}\right)\right)^{-p}$, then there is a connected component of $B(0, \delta)-N_{C \sigma}\left(w\left(Y_{l, C \sigma}\right)\right)$ of area more than $0.9 \pi \delta^{2}$. This is enough to finish the proof. The argument also applies in the parametric case, as shown in $[2]$.

\section{From matching paths to Lagrangian submanifolds}

Theorem 1.3 shows how to place a Lagrangian submanifold in special position with respect to a Lefschetz pencil. In the converse direction, it is natural to ask when it is possible to reconstruct a Lagrangian submanifold from a given Lefschetz pencil and a given path in the base $\mathbb{C P}^{1}$. We will restrict the discussion to the case of Lagrangian spheres, following arguments due to Donaldson and Seidel. Many of the ideas can be adapted for general Lagrangian submanifolds, but some technicalities are involved.

According to Theorem 1.3, a Lagrangian sphere can be realized as the lift of a path joining two critical points. But there may also be exotic spheres appearing in this way. To be precise, a compact smooth $n$-manifold admitting a Morse function with only one minimum and one maximum is the 
result of gluing two $n$-discs by a diffeomorphism of its boundary $S^{n-1}$. These manifolds are called twisted spheres (the twist is the gluing diffeomorphism of $\left.S^{n-1}\right)$. In particular, they are homotopy equivalent to the $n$-sphere. In dimensions $n \geq 5$, a result of Reeb [12] establishes that such a manifold is homeomorphic to the $n$-sphere.

In dimensions $n \geq 7$, all exotic spheres are twisted [15]. In dimensions $n \leq 6, n \neq 4$, there are no exotic spheres, so the only twisted sphere is $S^{n}$. In dimension $n=4$, there may be exotic spheres, but the only twisted sphere is the standard 4 -sphere. This is a result of Cerf [8].

Recall that, outside of the base points and critical points, a symplectic Lefschetz pencil carries a natural horizontal distribution given by the symplectic orthogonal to the fiber. Parallel transport along an arc $\gamma:[0,1] \rightarrow \mathbb{C P}^{1}$ which avoids the critical values induce a symplectomorphism between the smooth fibers above the endpoints. However, if one of the endpoints $\gamma(0)$ is a critical value, then one can associate to $\gamma$ a Lagrangian "vanishing disc" $D \subset M$ (also called "Lefschetz thimble"), which is the set of all the points in the fibers above $\gamma$ for which parallel transport converges to the critical point in the fiber above $\gamma(0)$. The boundary $S=\partial D$ is a Lagrangian sphere in the fiber above $\gamma(1)$, called the vanishing cycle associated to $\gamma$.

Vanishing cycles are actually framed Lagrangian spheres [13, Section 2]. Namely, the fact that $S$ bounds the disc $D$ determines a diffeomorphism $f: S^{n-1} \rightarrow S$ up to equivalence (two diffeomorphisms $f, f^{\prime}$ are said to be equivalent if $f^{-1} f^{\prime}$ is isotopic to an element of $\left.O(n) \subset \operatorname{Diff}\left(S^{n-1}\right)\right)$; for $n \geq 7$ the framing contains additional information. With this understood, we give the following

Definition 8.1. A matching path for a symplectic pencil $\phi$ is an embedded curve $\gamma:[0,1] \rightarrow \mathbb{C P}^{1}$ such that:

(i) the only critical values of the pencil which lie on the curve $\gamma$ are the endpoints $\gamma(0)$ and $\gamma(1)$;

(ii) the vanishing cycles $S_{0}, S_{1} \subset \phi^{-1}\left(\gamma\left(\frac{1}{2}\right)\right)$ associated with the arcs $\left.\gamma\right|_{[0,1 / 2]}$ and $\left.\gamma\right|_{[1 / 2,1]}$ are Hamiltonian isotopic as framed Lagrangian submanifolds of the fiber $\phi^{-1}\left(\gamma\left(\frac{1}{2}\right)\right)$ through a Hamiltonian isotopy with support away from the base point set.

If $S_{0}, S_{1}$ are Hamiltonian isotopic as Lagrangian submanifolds but may carry inequivalent framings, then we say that $\phi$ is a twisted matching path.

Recall that, viewing the framed Lagrangian spheres $S_{0}, S_{1}$ as the images of Lagrangian embeddings $i_{0}, i_{1}: S^{n-1} \rightarrow \phi^{-1}\left(\gamma\left(\frac{1}{2}\right)\right)$, we say that $S_{0}, S_{1}$ are Hamiltonian isotopic if $i_{0}, i_{1}$ are Hamiltonian isotopic Lagrangian embeddings up to composition by an element of $O(n)$.

A (twisted) matching path can be used to construct a smoothly embedded (twisted) $n$-sphere $S_{\gamma} \subset M$ in the following manner. Let $\left(\Psi_{s}\right)_{s \in[0,1]}$ be a 
Hamiltonian isotopy in the fiber $\phi^{-1}\left(\gamma\left(\frac{1}{2}\right)\right)$ connecting $S_{0}$ and $S_{1}$ (so $\Psi_{0}=\mathrm{Id}$ and $\left.\Psi_{1}\left(S_{0}\right)=S_{1}\right)$, and let $\chi:[0,1] \rightarrow[0,1]$ be a smooth function such that $\left.\chi\right|_{[0,1 / 3]}=0$ and $\left.\chi\right|_{[2 / 3,1]}=1$. For $t \in[0,1]$, denote by $\mathcal{P}_{t}: \phi^{-1}\left(\gamma\left(\frac{1}{2}\right)\right) \rightarrow$ $\phi^{-1}(\gamma(t))$ the map induced by parallel transport along $\gamma$. Then we let $S_{\gamma}=$ $\bigcup_{t \in[0,1]} \mathcal{P}_{t}\left(\Psi_{\chi(s)}\left(S_{0}\right)\right)$. It is easy to check that $S_{\gamma}$ is a standard $n$-sphere if and only if the framings of $\Psi_{1}\left(S_{0}\right)$ and $S_{1}$ are equivalent, i.e., if and only if $\gamma$ is a true matching path.

Although the (twisted) sphere $S_{\gamma}$ obtained in this way coincides with the vanishing discs $D_{0}$ and $D_{1}$ near its extremities, it is in general not Lagrangian (except if $S_{0}=S_{1}$, in which case we can choose $\Psi_{s}=$ Id and $S_{\gamma}=D_{0} \cup D_{1}$ is Lagrangian).

Remark 8.2. Any path isotopic to a (twisted) matching path through an isotopy fixing the endpoints in $\mathbb{C P}^{1}$ and avoiding the critical points is also a (twisted) matching path. This is because the symplectic connection associated to the pencil is Hamiltonian (i.e., parallel transport along contractible loops generates Hamiltonian isotopies).

Definition 8.3. Suppose that we have a family of Lefschetz pencils $\left\{\phi_{t}\right\}_{t \in[0,1]}$ and a family of paths $\gamma_{t} \subset \mathbb{C P}^{1}$ such that the endpoints of $\gamma_{t}$ are critical values of $\phi_{t}$. We say that the paths $\gamma_{0}$ and $\gamma_{1}$ are homotopic with respect to the family $\left\{\phi_{t}\right\}$ if the arcs $\gamma_{t}$ pass through critical values of $\phi_{t}$ only for a finite number of values of the parameter $t$, and if whenever $\gamma_{t_{j}}$ passes through a point $z_{j}=\gamma_{t_{j}}\left(s_{0}\right) \in \operatorname{crit}\left(\phi_{t_{j}}\right)$ with $0<s_{0}<1$, the vanishing cycles $S^{\prime}$ and $S^{\prime \prime}$ associated to the arcs $\left.\gamma_{t_{j}}\right|_{\left[0, s_{0} / 2\right]}$ and $\left.\gamma_{t_{j}}\right|_{\left[s_{0} / 2, s_{0}\right]}$ can be made mutually disjoint by compactly supported Hamiltonian isotopies inside $\phi_{t_{j}}^{-1}\left(\gamma_{t_{j}}\left(s_{0} / 2\right)\right)$.

Consider two homotopic paths $\gamma_{0}$ and $\gamma_{1}$, joined by a family $\gamma_{t}$ as in the definition. Assume that $\gamma_{0}$ is a (twisted) matching path: then $\gamma_{1}$ is also a (twisted) matching path. This can be seen by considering the families of vanishing cycles $S_{t, 0}, S_{t, 1} \subset \Sigma_{t}=\phi_{t}^{-1}\left(\gamma_{t}\left(\frac{1}{2}\right)\right), 0 \leq t \leq 1$. More precisely, to handle the situation where $\gamma_{t}$ passes through a critical value of $\phi_{t}$, we need to consider not just parallel transport but also Hamiltonian isotopies $\rho_{t}$ inside the fibers above $\gamma_{t}(\epsilon)$ and $\gamma_{t}(1-\epsilon)$ for some small $\epsilon>0 ; S_{t, 0}^{\prime}$ is then defined by taking parallel transport along $\left.\gamma_{t}\right|_{[0, \epsilon]}$, then applying $\rho_{t}$, and then parallel transport along $\left.\gamma_{t}\right|_{[\epsilon, 1 / 2]}$ (and similarly for $S_{t, 1}^{\prime}$ ). Definition 8.3 implies that, by choosing $\rho_{t}$ suitably, we can ensure that the perturbed vanishing disks avoid the critical values of $\phi_{t}$ for all $t$, and hence define Lagrangian spheres $S_{t, 0}^{\prime}, S_{t, 1}^{\prime}$ that depend continuously on $t$ and are Hamiltonian isotopic to the vanishing cycles for all $t$ (we can also assume $\rho_{0}=\rho_{1}=\mathrm{Id}$ ). With this understood, by assumption $S_{0,0}^{\prime}$ is isotopic to $S_{0,1}^{\prime}$, so $S_{t, 0}^{\prime}$ and $S_{t, 1}^{\prime}$ are mutually isotopic for all $t$, and even Hamiltonian isotopic because of exactness. In fact, we can find a family of exact symplectomorphisms identifying the 
symplectic submanifolds $\Sigma_{t}$ with each other, and the Hamiltonian isotopy between $S_{1,0}^{\prime}$ and $S_{1,1}^{\prime}$ can then be realized by juxtaposition of the families of exact Lagrangian spheres $\left(S_{1-t, 0}^{\prime}\right)_{0 \leq t \leq 1},\left(S_{0, s}^{\prime}\right)_{0 \leq s \leq 1}$, and $\left(S_{t, 1}^{\prime}\right)_{0 \leq t \leq 1}$.

So being a (twisted) matching path is a property that depends only on the relative homotopy type of the path and not on a particular realization.

The following lemma is an unpublished result of Donaldson. We thank Paul Seidel for communicating it to us. We give the parametric version for completeness.

Lemma 8.4. Let $\gamma_{t}$ be a 1-parametric family of (twisted) matching paths in a family of symplectic pencils $\phi_{t}, t \in[0,1]$ (of large enough degree). Then there exists a continuous family of Lagrangian (twisted) spheres $S_{t}$ in $M$ such that each of them is smoothly isotopic to $S_{\gamma_{t}}$.

Moreover, if for $j=0,1$ the vanishing cycles $S_{j, 0}$ and $S_{j, 1}$ coincide over the reference fiber, then the family of Lagrangian (twisted) spheres $S_{t}$ can be chosen in such a way that $S_{j}=S_{\gamma_{j}}$ for $j=0,1$.

Proof. We first assume that the isotopy does not cross critical points. Remove a fiber $\phi_{t}^{-1}\left(z_{t}\right)$ from each of the pencils $\phi_{t}$, where the point $z_{t}$ lies outside of the image of $\gamma_{t}$. After composition with a projective automorphism of $\mathbb{C P}^{1}=\mathbb{C} \cup\{\infty\}$, we can assume that $z_{t}=\infty$ and $\gamma_{t}(1 / 2)=0$. The fiber above infinity is the zero set of an asymptotically holomorphic section $s_{t}$, of which we may assume without loss of generality that it is transverse to zero provided $k$ is large enough (see [1] for details of how to get transversality in a generic fiber of a Donaldson pencil). From now on, we will consider the restrictions of the pencils $\phi_{t}$ to the open manifolds $M_{t}$, still denoted by $\phi_{t}: M_{t} \rightarrow \mathbb{C}$.

We are going to perturb the symplectic form in the fibers of $\phi_{t}$ over $\left.\gamma_{t}\right|_{[1 / 2-\epsilon, 1 / 2]}$, where $\epsilon>0$ is a small constant, in order to change the symplectic connection and make the two vanishing cycles match. After reparametrizing $\gamma_{t}$, we can assume that the interval over which we perturb the symplectic form is $[0,1]$ instead of $\left[\frac{1}{2}-\epsilon, \frac{1}{2}\right]$. Identify small closed neighborhoods of $\gamma_{t}([0,1])$ in $\mathbb{C P}^{1}$ with $D=[0,1] \times[-1,1]$, in such a way that $\gamma_{t}([0,1])$ is mapped to $[0,1] \times\{0\}$. Then we can construct a family of charts

$$
\Phi_{t}: D \times F \stackrel{\sim}{\longrightarrow} \phi_{t}^{-1}(D) \cap M_{t},
$$

where $F$ is an open symplectic (in fact Stein) fiber of $\phi_{t}$, by identifying symplectically all the fibers of $\phi_{t}$ above $D$ in such a way that parallel transport along $[0,1] \times\{0\}$ is horizontal. Hence, calling $(x, y)$ the coordinates on $D=[0,1] \times[-1,1]$,

$$
\Phi_{t}^{*}(\omega)=\sigma_{t}+\alpha_{t} \wedge d x+\beta_{t} \wedge d y+f_{t} d x \wedge d y,
$$

where $\sigma_{t}=\sigma$ is the pullback of a symplectic form on $F$, constant over $D$ and independent of $t$ (it follows from Moser's argument that the fibers of 
the various pencils $\phi_{t}$ are mutually symplectomorphic), while $\alpha_{t}(x, y)$ and $\beta_{t}(x, y)$ are 1 -forms in the fibers, with $\alpha_{t}(x, 0) \equiv 0$, and $f_{t}(x, y)$ is a positive function. The closedness of $\omega$ imposes the following relations:

$$
d \alpha_{t}=0, \quad d \beta_{t}=0, \quad d f_{t}=\frac{\partial \beta_{t}}{\partial x}-\frac{\partial \alpha_{t}}{\partial y},
$$

where the exterior differentials are only applied to the fiber directions (the $x$ and $y$ coordinates are treated as parameters). Choose a vector $v=\left(v_{1}, v_{2}\right) \in$ $T D$. The unique horizontal lifting of $v$ is a vector $\left(v, X_{v}\right)$, where $X_{v} \in T F$ is determined by the equation

$$
i_{X_{v}} \sigma=v_{1} \alpha_{t}+v_{2} \beta_{t} .
$$

In particular, the lifting of the segment $[0,1] \times\{0\}$ gives rise to a vector field with $X_{v}=0$.

Let $\Psi_{t, s}$ be a compactly supported Hamiltonian isotopy of the fiber $F$ sending the vanishing sphere $S_{t, 0}$ to $S_{t, 1}$ (recall that $t$ parametrizes the family and $s$ is the time parameter for the Hamiltonian flow of each member of the family). We want to change the symplectic connection (changing the symplectic form) in such a way that the horizontal lift of the segment $[0,1] \times\{0\}$ generates $\Psi_{t, s}$ instead of the identity map. Let $H_{t, s}$ be a family of Hamiltonian functions generating $\Psi_{t, s}$. Without loss of generality we may assume that $H_{t, s}$ vanishes identically for all $t \in[0, \delta] \cup[1-\delta, 1]$, for some small $\delta>0$. We define a function over $\left[\frac{1}{4}, \frac{3}{4}\right] \times\{0\} \times F \subset D \times F$ by the formula $\hat{F}_{t}(x, 0, p)=H_{t, 2(x-1 / 4)}(p)$. Extend $\hat{F}_{t}$ to all of $D \times F$ in such a way that it vanishes outside of $\left[\frac{1}{4}, \frac{3}{4}\right] \times\left[-\frac{1}{2}, \frac{1}{2}\right] \times F$. Define $\alpha_{t}^{\prime}=\alpha_{t}+d \hat{F}_{t}$ and $f_{t}^{\prime}=f_{t}-\frac{\partial \hat{F}_{t}}{\partial y}$, and consider the new 2 -form

$$
\omega_{t}^{\prime}=\sigma+\alpha_{t}^{\prime} \wedge d x+\beta_{t} \wedge d y+f_{t}^{\prime} d x \wedge d y .
$$

The closed 2-form $\omega_{t}^{\prime}=\Phi_{t}^{*}(\omega)+d\left(\hat{F}_{t} d x\right)$ coincides with $\Phi_{t}^{*}(\omega)$ in a neighborhood of the boundary of $F \times D$, therefore we can extend $\omega_{t}^{\prime}$ to a closed 2-form over $M$, coinciding with $\omega$ outside of $F \times D$. Moreover, by construction the horizontal lift of the segment $[0,1] \times\{0\}$ with respect to the symplectic connection induced by $\omega_{t}^{\prime}$ generates the flow $\Psi_{t, s}$, so that the vanishing cycles now coincide. In general, although $\omega_{t}^{\prime}$ is vertically non-degenerate, it need not be symplectic. We construct a new family of forms

$$
\tilde{\omega}_{t}=\omega_{t}^{\prime}+\phi_{t}^{*}\left(C_{t} d x \wedge d y\right),
$$

where $C_{t}$ is a positive real constant. For $C_{t}$ large enough the family $\tilde{\omega}_{t}$ is symplectic. Moreover, if $\Psi_{t, 0}=\Psi_{t, 1}=\mathrm{Id}$, then $\omega_{0}^{\prime}=\omega_{1}^{\prime}=\omega$ and the continuous family of constants $C_{t}$ can be chosen to satisfy $C_{0}=C_{1}=0$. Recall that $\tilde{\omega}_{t}$ and $\omega_{t}^{\prime}$ generate the same symplectic connection and therefore the (twisted) matching paths $\gamma_{t}$ generate Lagrangian (twisted) spheres $\tilde{S}_{t}$ for the symplectic structure $\tilde{\omega}_{t}$. 
There is a biparametric family of exact symplectic structures $\tilde{\omega}_{t, s}$ such that $\tilde{\omega}_{t, 0}=\tilde{\omega}_{t}$ and $\tilde{\omega}_{t, 1}=\omega$. To construct it we first shrink to zero the perturbation in $\alpha_{t}^{\prime}$ and then we shrink to zero the constant $C_{t}$ (the order is important to keep all the forms non-degenerate). Hence, each of these forms can be written as $\tilde{\omega}_{t, s}=\omega+a_{t, s} d\left(\hat{F}_{t} d x\right)+C_{t, s} \phi_{t}^{*}(d x \wedge d y)$, for some $a_{t, s}, C_{t, s} \geq 0$. By applying Moser's trick to the family of forms $\left\{\tilde{\omega}_{t, s}\right\}_{s \in[0,1]}$, we can find a family of diffeomorphisms $\psi_{t, s^{\prime}, s}$, defined over open subsets of $M_{t}$, such that $\psi_{t, s^{\prime}, s}\left(\tilde{\omega}_{t, s}\right)=\tilde{\omega}_{t, s+s^{\prime}}$. In the case where $\Psi_{0, s}=\Psi_{1, s}=\mathrm{Id}$, we have $\psi_{0, s^{\prime}, s}=\psi_{1, s^{\prime}, s}=$ Id. Now, we want to push-forward $\tilde{S}_{t}$ using the flow $\psi_{t, s^{\prime}, s}$ in order to obtain a family of Lagrangian (twisted) spheres for the initial symplectic structure $\omega$. However, since $M_{t}$ is an open manifold, $\psi_{t, s^{\prime}, s}$ is not necessarily well defined everywhere.

This difficulty can be avoided by using the exactness of the symplectic forms $\tilde{\omega}_{t, s}$ on $M_{t}$, and the existence of a Liouville vector field $v_{t, s}$ transverse to the boundary. More precisely, recall that over $M_{t}$ we can write $\omega=d \theta_{t}$, where $-k \theta_{t}$ is the imaginary part of the connection 1-form of $L^{\otimes k}$ in the trivialization given by the defining section $s_{t}$ of the fiber at infinity. In other terms, $\theta_{t}=-\frac{1}{k} \operatorname{Im}\left(s_{t}^{-1} \nabla s_{t}\right)$. The vector field $v_{t}$ such that $\omega\left(v_{t}, \cdot\right)=\theta_{t}$ is then a Liouville vector field for $\omega$, and since $s_{t}$ is asymptotically holomorphic and transverse to 0 , near the boundary of $M_{t}$ this vector field points everywhere outwards, in fact, near the boundary $v_{t}$ coincides up to $O\left(k^{-1 / 2}\right)$ with the gradient vector field of $-\frac{1}{k} \log \left|s_{t}\right|$. When we perturb the symplectic form to $\tilde{\omega}_{t, s}$ the existence of a Liouville vector field is preserved: we can write $\tilde{\omega}_{t, s}=d \tilde{\theta}_{t, s}$, where

$$
\tilde{\theta}_{t, s}=\theta_{t}+a_{t, s} \hat{F}_{t} d x+\frac{1}{2} C_{t, s} \phi_{t}^{*}(x d y-y d x) .
$$

The corresponding Liouville vector field $\tilde{v}_{t, s}$ (defined by $\left.\tilde{\omega}_{t, s}\left(\tilde{v}_{t, s}, \cdot\right)=\tilde{\theta}_{t, s}\right)$ is still pointing outwards near the boundary of $M_{t}$, as can be seen by checking that $\tilde{\theta}_{t, s} \wedge \tilde{\omega}_{t, s}$ has the required positivity property (here one uses the fact that the first perturbation term $\hat{F}_{t} d x$ is supported inside a compact subset of $M_{t}$ and, more importantly, the non-negativity of $C_{t, s}$ ).

Denote $k_{t, s, \tau}$ the flow generated by integrating $-\tilde{v}_{t, s}$ over a time $\tau$; this flow is well-defined everywhere and pushes inwards near the boundary of $M_{t}$. It is easy to construct two families of open sets $D_{t, 1} \subset D_{t, 2} \subset M_{t}$ with the following properties:

(i) $\tilde{S}_{t} \subset D_{t, 1}$.

(ii) There is a small $\epsilon>0$ (independent of $t$ and $s$ by compactness) for which $\psi_{t, \epsilon, s}\left(D_{t, 1}\right) \subset D_{t, 2} ;$ assume $l=\frac{1}{\epsilon} \in \mathbb{N}$.

(iii) There is a $\lambda_{t}>0$ such that $k_{t, s, \lambda_{t}}\left(D_{t, 2}\right) \subset D_{t, 1}$ for all $s, t$.

In the case where $\Psi_{0, s}=\Psi_{1, s}=\mathrm{Id}$, we can choose $D_{j, 1}=D_{j, 2}$ and $\lambda_{j}=0$ for $j \in\{0,1\}$. 
Observe that the flow $k_{t, s, \lambda_{t}}$ preserves the property of being Lagrangian with respect to $\tilde{\omega}_{t, s}$. Therefore, we can proceed as follows. Start with $\tilde{S}_{t, 0}=\tilde{S}_{t}$, and let $\tilde{S}_{t, 1}=k_{t, \epsilon, \lambda_{t}} \circ \psi_{t, \epsilon, 0}\left(\tilde{S}_{t, 0}\right)$. By construction $\tilde{S}_{t, 1} \subset D_{t, 1}$. We can then repeat the process, defining

$$
\tilde{S}_{t, j+1}=k_{t,(j+1) \epsilon, \lambda_{t}} \circ \psi_{t, \epsilon, j \epsilon}\left(\tilde{S}_{t, j}\right) .
$$

At the end we get a family of Lagrangian (twisted) spheres $\tilde{S}_{t, l}$ with respect to the initial symplectic structure $\omega$, which completes the proof in the case where the isotopy does not cross any critical point of $\phi_{t}$.

In the general case, the argument remains the same. The only important observation is that, by definition, suitably chosen Hamiltonian isotopies in the fibers can be used to ensure that the spheres we consider stay away from the vanishing cycles at the critical points hit by the family of (twisted) matching paths $\gamma_{t}$. Hence, we can still find a family of Hamiltonian isotopies $\Psi_{t, s}$ in the fibers of $\phi_{t}$, sending the vanishing sphere $S_{t, 0}$ to $S_{t, 1}$, and with support in a compact subset of $M_{t}$ disjoint from the support of the generalized Dehn twists arising as monodromies around the different critical values encountered by $\gamma_{t}$.

Remark 8.5. The above construction does not provide a canonical (twisted) sphere associated to the (twisted) matching path. However, all the possible choices are Lagrangian (and hence Hamiltonian) isotopic. This is because the spaces of choices that appear in the proof are all path connected.

\section{Isotopies of Lagrangian submanifolds}

The above results allow us to tentatively identify Lagrangian spheres (resp. twisted spheres) with matching paths (resp. twisted matching paths). By Theorem 1.3 we know that, given any Lagrangian (twisted) sphere, there exists a pencil for which it fibers above a (twisted) matching path. Conversely, by Lemma 8.4 we know that a (twisted) matching path gives rise to a Lagrangian (twisted) sphere up to isotopy. It is therefore natural to look for a general result identifying homotopy classes of (twisted) matching paths and Lagrangian (twisted) spheres. For this purpose, we now study more carefully the behavior of our construction in the presence of isotopic Lagrangian (twisted) spheres (which will correspond to homotopic paths, cf. Remark 8.2).

Consider a fixed sequence of Donaldson pencils $\phi_{k}^{0}$, and introduce the following

Definition 9.1. A sequence of (twisted) matching paths $\gamma_{k}$ for the sequence of Donaldson pencils $\phi_{k}^{0}$, associated to Lagrangian (twisted) spheres in the same Hamiltonian isotopy class for all $k$, is called asymptotically stable if for large $k$ there exists a family $\left(\phi_{k, t}\right)_{0 \leq t \leq 1}$ of Donaldson pencils with $\phi_{k}^{0}=\phi_{k, 0}$, 
such that the path $\gamma_{k}$ is homotopic via the family $\phi_{k, t}$ to the path

$$
\begin{aligned}
\hat{\gamma}_{k}:[0,1] & \longrightarrow \mathbb{C P}^{1} \\
t & \longrightarrow e^{2 i k^{1 / 2} t+k^{-1 / 2} t}
\end{aligned}
$$

and the Lagrangian (twisted) sphere associated to the (twisted) matching path $\hat{\gamma}_{k}$ arises from the construction described in the proof of Theorem 1.3 (this implies in particular a number of transversality conditions on $\phi_{k, 1}$ near the Lagrangian (twisted) sphere).

Remark 9.2. The sequences generated by Theorem 1.3 are asymptotically stable sequences once we isotope the resulting family of pencils $\phi_{k}$ to the fixed family $\phi_{k}^{0}$. In fact, these are the only computable examples.

The following result is a direct corollary of Theorem 1.4 and Definition 9.1:

Theorem 9.3. Let $S_{0}$ and $S_{1}$ be two Hamiltonian isotopic Lagrangian (twisted) spheres, associated to asymptotically stable sequences of (twisted) matching paths $\gamma_{k, 0}$ and $\gamma_{k, 1}$ in the pencils $\phi_{k}^{0}$. Then, for large enough $k$, there exists a family of Lefschetz pencils $\left(\phi_{k, t}\right)_{0 \leq t \leq 1}$, with $\phi_{k, 0}=\phi_{k, 1}=\phi_{k}^{0}$, such that the (twisted) matching path $\gamma_{k, 0}$ is homotopic to $\gamma_{k, 1}$ via the family $\phi_{k, t}$.

Considering all asymptotically stable sequences of matching paths, define $\mathcal{M}=\left\{\left[\gamma_{k}\right]: \gamma_{k}\right.$ is an asymptotically stable sequence for $\phi_{k}^{0}, k$ large enough $\}$, where $\left[\gamma_{k}\right]$ denotes the homotopy class of the matching path $\gamma_{k}$. Define similarly a set $\mathcal{M}^{t}$ by considering asymptotically stable sequences of twisted matching paths up to homotopy. Define the set

$$
\text { Pen }=\left\{\left\{\phi_{k}\right\}: \phi_{k} \text { is a sequence of Donaldson pencils }\right\} .
$$

There is a natural action

$$
\pi_{1}\left(\mathrm{Pen},\left\{\phi_{k}^{0}\right\}\right) \times \mathcal{M} \longrightarrow \mathcal{M},
$$

defined by transport along a family of pencils (and similarly for $\mathcal{M}^{t}$ ). Then we have:

Theorem 9.4. The set of Hamiltonian isotopy classes of Lagrangian spheres (resp. twisted spheres) in the symplectic manifold $(M, \omega)$ is in one-to-one correspondence with the set $\mathcal{M} / \pi_{1}\left(\operatorname{Pen},\left\{\phi_{k}^{0}\right\}\right)$ (resp. $\mathcal{M}^{t} / \pi_{1}\left(\operatorname{Pen},\left\{\phi_{k}^{0}\right\}\right)$ ).

In principle, this result reduces (at least in dimension 4) the problem of the classification of Lagrangian spheres in a symplectic manifold to the purely algebro-combinatorial problem of classifying matching paths in a sequence of pencils. However, the notion of asymptotic stability of matching paths is a very unnatural one and makes things much less practical.

To eliminate this requirement, and to simplify the discussion, one should consider the behavior of pencils and matching paths under degree doubling, 
i.e., upon passing from sections of $L^{\otimes k}$ to sections of $L^{\otimes 2 k}$. In general, this requires an understanding of the "degree doubling" process (see, e.g., $[\mathbf{7}, \mathbf{1 6}])$. For simplicity, we restrict ourselves to the algebraic case, where estimated transversality is not needed. Recall that, in that case, there is a natural way of inducing a sequence of matching paths in a sequence $\phi_{k}^{0}$ of pencils $\left(k=2^{l} k_{0}, l \in \mathbb{N}\right)$, built as follows: starting from a given pencil $\phi_{k}^{0}=s_{k}^{1} / s_{k}^{2}$, construct a new pencil $\phi_{2 k}^{0}=s_{2 k}^{1} / s_{2 k}^{2}$ whose defining sections are arbitrarily small generic perturbations of $\sigma_{2 k}^{1}=s_{k}^{1} \otimes s_{k}^{1}$ and $\sigma_{2 k}^{2}=s_{k}^{1} \otimes s_{k}^{2}$. Repeating the process we get a sequence of pencils $\phi_{k}^{0}, k=k_{0} 2^{l}$. It is easy to check that the set of critical values of $\phi_{k}^{0}$ identifies naturally with a subset of the critical values of $\phi_{2 k}^{0}$. More precisely, there is a diffeomorphism from an open ball $B(0, R)$ of $\mathbb{C P}^{1}$ to itself which takes the critical values of $\phi_{k}^{0}$ to critical values of $\phi_{2 k}^{0}$, and such that the rest of the critical values of $\phi_{2 k}^{0}$ remain outside that ball; in fact, the monodromy of $\phi_{k}^{0}$ naturally "embeds" into that of $\phi_{2 k}^{0}[\mathbf{7}, \mathbf{1 6}]$. This makes it possible to build from a matching path $\gamma_{k_{0}}$ for $\phi_{k_{0}}^{0}$ a sequence of matching paths $\gamma_{k}$ for $\phi_{k}^{0}$, for all $k=2^{l} k_{0}$. It is easy to check that the Lagrangian sphere associated to each element of the family is always the same up to Hamiltonian isotopy. We will call "natural sequence" the sequence of matching paths obtained in this manner from a given matching path. Then Theorem 9.4 can be reformulated as

Theorem 9.5. The set of natural sequences of (twisted) matching paths in a family of Lefschetz pencils $\phi_{k}^{0}$ on a projective manifold up to the action of $\pi_{1}\left(\mathrm{Pen},\left\{\phi_{k}^{0}\right\}\right)$ is in bijection with the set of Hamiltonian isotopy classes of Lagrangian (twisted) spheres.

\section{Pencil automorphisms, symplectomorphisms, and matching paths}

In this section, we discuss the connection between automorphisms of the monodromy data of a Lefschetz pencil and symplectomorphisms of the total space of the pencil; most of the discussion follows ideas of Donaldson and Seidel (see in particular Section (1d) of [14]).

10.1. The group of pencil automorphisms. To any Lefschetz pencil one can attach a group of "pencil automorphisms", which can be viewed either geometrically (a pencil automorphism is then a homeomorphism which lifts to a diffeomorphism of the corresponding Lefschetz fibration on the blownup manifold, mapping fibers to fibers and inducing fiberwise symplectomorphisms), or (taking isotopy classes) combinatorially in terms of monodromy data. We will adopt the combinatorial point of view here.

Recall that the monodromy of a symplectic Lefschetz pencil $\phi: M-N \rightarrow$ $\mathbb{C P}^{1}$ is defined by fixing a base point $\alpha_{0} \in \mathbb{C P}^{1}$ and considering the isotopy classes of the symplectomorphisms of the reference fiber $\Sigma_{0}=\overline{\phi^{-1}\left(\alpha_{0}\right)}$ 
induced by parallel transport along loops in $\mathbb{C P}^{1}-\operatorname{crit}(\phi)$. The monodromy morphism takes values in the symplectic mapping class group of the fiber relatively to the base points. More precisely, let

$\operatorname{Map}\left(\Sigma_{0}, N\right)=\pi_{0}\left\{g \in \operatorname{Symp}\left(\Sigma_{0}, \omega_{\mid \Sigma_{0}}\right) \mid \forall p \in N, g(p)=p\right.$ and $\left.d g(p)=\operatorname{Id}\right\}$.

Then, after removing a neighborhood of a smooth fiber $\Sigma_{\infty}$ of the pencil, we obtain a fibration over a large disc $\Delta$ containing all the critical values of $\phi$, whose monodromy is given by a morphism

$$
\theta: \pi_{1}\left(\Delta-\operatorname{crit}(\phi), \alpha_{0}\right) \longrightarrow \operatorname{Map}\left(\Sigma_{0}, N\right) .
$$

Let $r$ be the number of critical points of $\phi$, which we assume to all lie in distinct fibers, and let $\mathcal{A}=\operatorname{crit}(\phi) \subset \Delta \subset \mathbb{C P}^{1}$ be the set of critical values. For simplicity, we assume that the base point $\alpha_{0}$ lies on the boundary of $\Delta$. Choosing a set of geometric generators $\gamma_{1}, \ldots, \gamma_{r}$ of $\pi_{1}\left(\Delta-\mathcal{A}, \alpha_{0}\right)$ (each encircling one of the critical values), each $\theta\left(\gamma_{i}\right)$ is a Dehn twist about an (exact) Lagrangian sphere $L_{i} \subset \Sigma_{0}-N$ (the vanishing cycle associated to $\left.\gamma_{i}\right)$. Any loop in $\pi_{1}\left(\Delta-\mathcal{A}, \alpha_{0}\right)$ that bounds a disc containing exactly one critical value can be written in the form $g^{-1} \gamma_{i} g$ and is mapped by $\theta$ to a Dehn twist about the Lagrangian sphere $\theta(g)\left(L_{i}\right)$. Recall moreover from Section 8 that each vanishing cycle carries a natural framing, i.e., a parametrization (up to equivalence) as a Lagrangian embedding of $S^{n-1}$ into $\Sigma_{0}$.

Definition 10.1. Let $\hat{\mathcal{M}}$ be the set of all pairs $\left(\zeta, L_{\zeta}\right)$ where $\zeta: \pi_{1}(\Delta-$ $\left.\mathcal{A}, \alpha_{0}\right) \rightarrow \operatorname{Map}\left(\Sigma_{0}, N\right)$ is a group homomorphism and $L_{\zeta}$ is a map from the set $\Pi$ of all conjugates of geometric generators in $\pi_{1}\left(\Delta-\mathcal{A}, \alpha_{0}\right)$ to the set of Hamiltonian isotopy classes of (exact) framed Lagrangian spheres in $\Sigma_{0}-N$, such that: $(i) \forall \gamma \in \Pi, \zeta(\gamma)$ is the Dehn twist about $L_{\zeta}(\gamma) ;(i i)$ $\forall \gamma \in \Pi, \forall g \in \pi_{1}\left(\Delta-\mathcal{A}, \alpha_{0}\right), L_{\zeta}\left(g^{-1} \gamma g\right)=\zeta(g)\left(L_{\zeta}(\gamma)\right)$.

Then we can associate to the Lefschetz pencil $\phi$ an element $\hat{\theta}=\left(\theta, L_{\theta}\right) \in$ $\hat{\mathcal{M}}$, where $\theta$ is the monodromy morphism and $L_{\theta}$ is characterized by the property that $L_{\theta}\left(\gamma_{i}\right)=\left[L_{i}\right]$. When $\operatorname{dim} M=4$, one can recover the vanishing cycles from the monodromy morphism (using the exactness property to determine the Hamiltonian isotopy class), so in that case $\hat{\theta}$ contains no more information than $\theta$; in higher dimensions it is unknown whether $\theta$ determines $\hat{\theta}$.

It is a result of Gompf $[\mathbf{1 1}]$ that the "enhanced" monodromy morphism $\hat{\theta}$ determines the topology of the Lefschetz pencil over a large disc containing all the critical values of $\phi$, and up to a choice in $\pi_{1} \operatorname{Symp}\left(\Sigma_{0}, N\right)$ (describing the attaching map near the fiber at infinity), the symplectic manifold $(M, \omega)$ up to symplectic isotopy. This result relies on the fact that the fibers of the pencil are Poincaré dual to a multiple of $[\omega]$ : in general, applying Thurston's argument to a Lefschetz fibration yields symplectic forms that are only canonical up to deformation equivalence, but in the case of a pencil, 
after blowing down the exceptional divisor all these forms become isotopic up to scaling by a constant factor.

We will also consider the group

$$
\mathcal{B}\left(\Sigma_{0}, N\right)=\pi_{0}\left\{g \in \operatorname{Symp}\left(\Sigma_{0}, \omega_{\mid \Sigma_{0}}\right) \mid g(N)=N\right\} .
$$

This group acts on $\operatorname{Map}\left(\Sigma_{0}, N\right)$ by conjugation, and given an element $g \in$ $\mathcal{B}\left(\Sigma_{0}, N\right)$ we denote by $g_{*}$ the corresponding automorphism of $\operatorname{Map}\left(\Sigma_{0}, N\right)$. Combining this with the natural action of $\mathcal{B}\left(\Sigma_{0}, N\right)$ on the set of isotopy classes of framed Lagrangian spheres, we obtain an action of $\mathcal{B}\left(\Sigma_{0}, N\right)$ on $\hat{\mathcal{M}}$, given by $g_{*}:\left(\zeta, L_{\zeta}\right) \mapsto\left(g_{*} \circ \zeta, g \circ L_{\zeta}\right)$.

When $M$ is a 4 -manifold, let $h$ be the genus of $\Sigma_{0}$ and $n$ the number of base points: then $\operatorname{Map}\left(\Sigma_{0}, N\right)=\operatorname{Map}_{h, n}$ is the mapping class group of a genus $h$ surface with $n$ boundary components, and $\mathcal{B}\left(\Sigma_{0}, N\right)=\mathcal{B}_{h, n}$ is the braid group on $n$ strings on a genus $h$ surface.

Finally, denote by $B_{r}$ the classical braid group on $r$ strings, which can be viewed either as the fundamental group of the configuration space of $r$ distinct points in the $\operatorname{disc}\left(B_{r}=\pi_{1}\left(\operatorname{Conf}_{r}(\Delta), \mathcal{A}\right)\right)$ or in terms of isotopy classes of compactly supported orientation-preserving diffeomorphisms of $\Delta$ mapping $\mathcal{A}$ to itself. The latter description lets us associate to any braid $b \in B_{r}$ an automorphism $b_{*}$ of $\pi_{1}\left(\Delta-\mathcal{A}, \alpha_{0}\right)$. Hence there is a natural right action of $B_{r}$ on $\hat{\mathcal{M}}$ (by composition). With the above notations, we can make the following definition:

Definition 10.2. The group of combinatorial automorphisms of $\phi$ is

$$
\Gamma(\phi)=\left\{(b, g) \in B_{r} \times \mathcal{B}\left(\Sigma_{0}, N\right) \mid \hat{\theta} \circ b_{*}=g_{*}(\hat{\theta})\right\} .
$$

In other words, $\Gamma(\phi)$ is the stabilizer of the monodromy data $\hat{\theta} \in \hat{\mathcal{M}}$ for the natural actions of the braid group (by Hurwitz moves) and automorphisms of the fiber (by conjugation).

There is a natural homomorphism $\rho: \Gamma(\phi) \rightarrow \pi_{0} \operatorname{Symp}(M, \omega)$, which can be described as follows. Given an element $(b, g) \in \Gamma(\phi)$, choose a geometric representative of the braid $b$ inside a cylinder $\Delta \times[0,1] \subset \mathbb{C P}^{1} \times[0,1]$, and use it to build a 1-parameter family of Lefschetz pencils $\phi_{t}$ (each having the same total space $M$ and the same monodromy, but with critical values depending on $t \in[0,1]$ as prescribed by the braid $b$ ). By identifying the two ends of the cylinder, we can close the braid $b$ and obtain a link in $\Delta \times S^{1} \subset \mathbb{C P}^{1} \times S^{1}$. The equality $\hat{\theta} \circ b_{*}=g_{*}(\hat{\theta})$ makes it possible to identify the Lefschetz pencils $\phi_{0}=$ $\phi$ and $\phi_{1}$ by means of a fiberwise symplectomorphism in the isotopy class $g \in \mathcal{B}\left(\Sigma_{0}, N\right)$, up to a symplectic isotopy (using Gompf's characterization of symplectic structures on Lefschetz pencils [11]). This yields a family of symplectic Lefschetz pencils parametrized by elements of $S^{1}$, whose total space $W$ carries a structure of symplectic fiber bundle $(M, \omega) \rightarrow W \rightarrow S^{1}$; its monodromy is the element of $\pi_{0} \operatorname{Symp}(M, \omega)$ naturally associated to the automorphism $(b, g)$. 
The following asymptotic surjectivity result is a direct consequence of Donaldson's result of uniqueness up to isotopy [10]:

Proposition 10.3. Let $\phi_{k}: M-N_{k} \rightarrow \mathbb{C P}^{1}, k \gg 0$ be a sequence of Donaldson's symplectic Lefschetz pencils. Then for every $\eta \in \pi_{0} \operatorname{Symp}(M, \omega)$ there exists an integer $k(\eta)$ such that $\eta$ belongs to the image of the natural homomorphism $\rho: \Gamma\left(\phi_{k}\right) \rightarrow \pi_{0} \operatorname{Symp}(M, \omega)$ for all $k \geq k(\eta)$.

Proof. Let $\tilde{\eta}$ be a symplectomorphism in the isotopy class $\eta$, and equip $M$ with $\omega$-compatible almost-complex structures $\left(J_{t}\right)_{t \in[0,1]}$ such that $J_{0}=$ $\tilde{\eta}_{*}\left(J_{1}\right)$. By identifying the boundaries of $M \times[0,1]$ via the symplectomorphism $\tilde{\eta}$, we can build a symplectic fiber bundle over $S^{1}$ with fiber $(M, \omega)$ and monodromy $\eta$. Donaldson's construction of Lefschetz pencils $[\mathbf{1 0}]$ applies to this 1-parameter family and provides, for $k \gg 0$, a family of symplectic Lefschetz pencils $\tilde{\phi}_{k, t}: M-N_{k, t} \rightarrow \mathbb{C P}^{1}$ such that $\tilde{\phi}_{k, 1}=\tilde{\phi}_{k, 0} \circ \tilde{\eta}$ (in [10] the case where the parameter is in $t \in[0,1]$ is used to prove uniqueness up to isotopy; the case where the parameter belongs to $S^{1}$ is exactly identical). We can also easily require the critical values of $\tilde{\phi}_{k, t}$ to remain distinct for all values of $t$. The monodromy of the family $\tilde{\phi}_{k, t}$ can then naturally be expressed as an element $(b, g) \in \Gamma\left(\tilde{\phi}_{k, 0}\right)$ by considering the motion of the critical values as $t$ varies in $S^{1}$ (which gives the braid $b$ ) and the isotopy class of the induced symplectomorphism of a generic fiber (which gives $g$ ); by construction we have $\rho(b, g)=\eta$. Finally, it is known that the Donaldson pencils $\tilde{\phi}_{k, 0}$ and $\phi_{k}$ are mutually isotopic for large enough $k[\mathbf{1 0}]$, so that we can naturally identify $\Gamma\left(\tilde{\phi}_{k, 0}\right)$ with $\Gamma\left(\phi_{k}\right)$.

It is an interesting question to ask whether there is a value of $k$ for which the morphism $\rho$ is surjective. A positive answer might follow from a better understanding of the behavior of the group of pencil automorphisms under stabilization by degree doubling.

10.2. Matching paths, Dehn twists, and trivial automorphisms. Matching paths provide an explicit way to view Dehn twists along Lagrangian spheres in $M$ in this context. We begin with some terminology. Let $\delta:[0,1] \rightarrow \Delta \subset \mathbb{C P}^{1}$ be an embedded arc meeting $\mathcal{A}$ only at its endpoints $\delta(0)$ and $\delta(1)$. Then the positive half-twist along $\delta$ is the braid $\sigma_{\delta} \in B_{r}$ which exchanges the two points $\delta(0)$ and $\delta(1)$ by a 180-degree counterclockwise rotation in a small neighborhood of $\delta([0,1])$. Also, choose an arc $\eta$ in $\Delta-(\mathcal{A} \cup \delta)$ joining the base point $\alpha_{0}$ to the point $\delta(0)$; let $\eta^{\prime}$ be the oriented boundary of a small tubular neighborhood of $\eta$, and let $\eta^{\prime \prime}$ be the image of $\eta^{\prime}$ by the half-twist $\sigma_{\delta}$. Then $\eta^{\prime}, \eta^{\prime \prime} \in \Pi$ are conjugates of geometric generators of $\pi_{1}\left(\Delta-\mathcal{A}, \alpha_{0}\right)$; the pair $\left(\eta^{\prime}, \eta^{\prime \prime}\right)$ is called a supporting pair for the arc $\delta$, and is unique up to simultaneous conjugation $\left(\eta^{\prime}, \eta^{\prime \prime}\right) \mapsto\left(g^{-1} \eta^{\prime} g, g^{-1} \eta^{\prime \prime} g\right)$ in $\pi_{1}\left(\Delta-\mathcal{A}, \alpha_{0}\right)$. In particular, the pair of vanishing cycles $\left(L_{\theta}\left(\eta^{\prime}\right), L_{\theta}\left(\eta^{\prime \prime}\right)\right)$ is 
uniquely determined up to simultaneous action of an automorphism in the monodromy group $\left(L_{\theta}\left(\eta^{\prime}\right), L_{\theta}\left(\eta^{\prime \prime}\right)\right) \mapsto\left(\theta(g)\left(L_{\theta}\left(\eta^{\prime}\right)\right), \theta(g)\left(L_{\theta}\left(\eta^{\prime \prime}\right)\right)\right)$.

Proposition 10.4. Let $\gamma:[0,1] \rightarrow \Delta \subset \mathbb{C P}^{1}$ be a matching path for the Lefschetz pencil $\phi$, corresponding to a Lagrangian sphere $S_{\gamma} \subset M$. Then $\left(\sigma_{\gamma}, 1\right) \in \Gamma(\phi)$, and $\rho\left(\sigma_{\gamma}, 1\right)=\left[\tau_{S_{\gamma}}\right]$, where $\tau_{S_{\gamma}}$ is the Dehn twist along $S_{\gamma}$.

Proof. Let $\left(\eta^{\prime}, \eta^{\prime \prime}\right)$ be a supporting pair for $\gamma$. By choosing geometric generators for the complement of $(\eta \cup \gamma)$ in $\Delta$, we can complete the pair $\left(\eta^{\prime}, \eta^{\prime \prime}\right)$ to an ordered collection of generators $\left(\gamma_{i}\right)_{1 \leq i \leq r}$ of $\pi_{1}\left(\Delta-\mathcal{A}, \alpha_{0}\right)$ such that $\gamma_{i} \cap \gamma=\emptyset$ for all $i \geq 3$. Then the action of $\sigma_{\gamma}$ on $\pi_{1}\left(\Delta-\mathcal{A}, \alpha_{0}\right)$ is given by $\sigma_{\gamma}\left(\eta^{\prime}\right)=\eta^{\prime \prime}, \sigma_{\gamma}\left(\eta^{\prime \prime}\right)=\eta^{\prime \prime} \eta^{\prime}\left(\eta^{\prime \prime}\right)^{-1}$, and $\sigma_{\gamma}\left(\gamma_{i}\right)=\gamma_{i}$ for all $i \geq 3$.

By definition of a matching path, the vanishing cycles associated to $\eta^{\prime}$ and $\eta^{\prime \prime}$ are Hamiltonian isotopic as framed Lagrangian submanifolds, i.e., $L_{\theta}\left(\eta^{\prime}\right)=L_{\theta}\left(\eta^{\prime \prime}\right)$. This implies immediately that $\hat{\theta} \circ\left(\sigma_{\gamma}\right)_{*}=\hat{\theta}$, i.e., $\left(\sigma_{\gamma}, 1\right)$ is an automorphism of the pencil.

Construct a family of pencils $\left(\phi_{t}\right)$ whose monodromy is $g=\rho\left(\sigma_{\gamma}, 1\right)$ by choosing a representative of the braid $\sigma_{\gamma}$ in $\Delta \times[0,1]$ and identifying the two ends of the cylinder $t=0$ and $t=1$, as explained above. Because the braid $\sigma_{\gamma}$ has a representative supported in a small neighborhood $U_{\gamma}$ of the matching path $\gamma$, and because $\phi$ is trivial over $U_{\gamma}$ away from the vanishing cycle, it is easy to see that the isotopy class $g$ admits a representative which coincides with Id outside of a small neighborhood of the sphere $S_{\gamma}$. The argument can therefore be completed by considering a universal local model for the family of pencils $\phi_{t}$ over a neighborhood of $S_{\gamma}$. The existence of such a standard model relies on the fact that the framings of the vanishing cycles at both extremities of $\gamma$ are isotopic, which allows us to patch together the local models for the two critical points of $\phi$.

Consider the map $F=\left(F_{1}, F_{2}\right): \mathbb{C}^{n+1} \rightarrow \mathbb{C}^{2}$ defined by

$$
F\left(z_{1}, \ldots, z_{n+1}\right)=\left(z_{1}^{2}+\ldots+z_{n+1}^{2}, z_{n+1}\right) .
$$

For $t=\epsilon \mathrm{e}^{i \theta}$, the restriction of $F$ to the hypersurface $X_{t}=F_{1}^{-1}(t)=\left\{z_{1}^{2}+\right.$ $\left.\cdots+z_{n+1}^{2}=t\right\}$ induces a Lefschetz fibration $F_{2}: X_{t} \rightarrow \mathbb{C}$, whose generic fiber $F_{2}^{-1}(u)$ is a smooth quadric in $\mathbb{C}^{n} \times\{u\}$, defined by the equation $z_{1}^{2}+\cdots+z_{n}^{2}=t-u^{2}$. There are two singular fibers, and the corresponding critical values are the two square roots of $t$. The straight line segment between the two critical values $\pm t^{1 / 2}$ is a matching path for $F_{2}$, and the corresponding Lagrangian sphere is $S_{t}=X_{t} \cap\left(\mathrm{e}^{i \theta / 2} \mathbb{R}\right)^{n+1}$. As $\theta$ varies from 0 to $2 \pi$, the two critical values of $F_{2}$ are exchanged by a half-twist $\sigma$ along the matching path.

On the other hand, $F_{1}: \mathbb{C}^{n+1} \rightarrow \mathbb{C}$ provides a local model for a neighborhood of a critical point of a Lefschetz fibration in complex dimension $n+1$. Therefore, the monodromy of the family of symplectic manifolds $X_{t=\epsilon \exp (i \theta)}$ 
as $\theta$ varies from 0 to $2 \pi$ is the Dehn twist along the vanishing cycle associated to the singular fiber $X_{0}$ of $F_{1}$; this vanishing cycle is precisely the Lagrangian sphere $X_{\epsilon} \cap \mathbb{R}^{n+1}=S_{\epsilon}$, and so we have $\rho(\sigma, 1)=\left[\tau_{S_{\epsilon}}\right]$.

It is also possible to construct explicitly many elements in the kernel of the morphism $\rho: \Gamma(\phi) \rightarrow \pi_{0} \operatorname{Symp}(M, \omega)$ (i.e., "trivial" pencil automorphisms). Let $\gamma:[0,1] \rightarrow \Delta$ be an embedded arc with endpoints in $\mathcal{A}$, choose a supporting pair $\left(\eta^{\prime}, \eta^{\prime \prime}\right)$, and let $S^{\prime}, S^{\prime \prime} \subset \Sigma_{0}$ be Lagrangian spheres in the isotopy classes $L_{\theta}\left(\eta^{\prime}\right)$ and $L_{\theta}\left(\eta^{\prime \prime}\right)$.

Proposition 10.5. (a) If $S^{\prime}$ and $S^{\prime \prime}$ are disjoint, then $\left(\sigma_{\gamma}^{2}, 1\right) \in \Gamma(\phi)$ and $\rho\left(\sigma_{\gamma}^{2}, 1\right)=1$.

(b) If $S^{\prime}$ and $S^{\prime \prime}$ intersect transversely in a single point, then $\left(\sigma_{\gamma}^{3}, 1\right) \in \Gamma(\phi)$ and $\rho\left(\sigma_{\gamma}^{3}, 1\right)=1$.

Proof. The structure of the argument is the same as for the previous proposition. We start by completing $\left(\eta^{\prime}, \eta^{\prime \prime}\right)$ to an ordered collection of generators $\left(\gamma_{i}\right)_{1 \leq i \leq r}$ of $\pi_{1}\left(\Delta-\mathcal{A}, \alpha_{0}\right)$ such that $\gamma_{i} \cap \gamma=\emptyset$ for $i \geq 3$. In the case where $S^{\prime}$ and $S^{\prime \prime}$ are disjoint, observe that $\sigma_{\gamma}^{2}$ maps $\eta^{\prime}$ to $\tilde{\eta}^{\prime}=\eta^{\prime \prime} \eta^{\prime}\left(\eta^{\prime \prime}\right)^{-1}$, and $\eta^{\prime \prime}$ to $\tilde{\eta}^{\prime \prime}=\eta^{\prime \prime} \eta^{\prime} \eta^{\prime \prime}\left(\eta^{\prime}\right)^{-1}\left(\eta^{\prime \prime}\right)^{-1}$, while the other generators are preserved. Since $\theta\left(\eta^{\prime \prime}\right)=\left[\tau_{S^{\prime \prime}}\right]$ has a representative supported in a neighborhood of $S^{\prime \prime}$, it acts trivially on $S^{\prime}$. Therefore $L_{\theta}\left(\tilde{\eta}^{\prime}\right)=\theta\left(\eta^{\prime \prime}\right)^{-1}\left(L_{\theta}\left(\eta^{\prime}\right)\right)=L_{\theta}\left(\eta^{\prime}\right)$, and similarly $L_{\theta}\left(\tilde{\eta}^{\prime \prime}\right)=\theta\left(\eta^{\prime \prime}\right)^{-1}\left(\theta\left(\eta^{\prime}\right)^{-1}\left(L_{\theta}\left(\eta^{\prime \prime}\right)\right)\right)=L_{\theta}\left(\eta^{\prime \prime}\right)$, which implies that $\left(\sigma_{\gamma}^{2}, 1\right) \in \Gamma(\phi)$.

To see that the isotopy class $\rho\left(\sigma_{\gamma}^{2}, 1\right)$ is trivial, observe that the automorphism $\left(\sigma_{\gamma}^{2}, 1\right)$ corresponds to the monodromy of a $S^{1}$-family of pencils where two of the critical values simply move around each other. Because the two vanishing cycles $S^{\prime}$ and $S^{\prime \prime}$ are mutually disjoint, this $S^{1}$-family of pencils bounds a $D^{2}$-family of symplectic Lefschetz pencils on $M$ (one of which has two critical points in the same fiber, but this is not a problem since the vanishing cycles are disjoint). This implies that the monodromy is trivial, i.e., $\rho\left(\sigma_{\gamma}^{2}, 1\right)=1$.

In the case where $S^{\prime}$ and $S^{\prime \prime}$ intersect transversely in a single point, we consider the action of $\sigma_{\gamma}^{3}$ on $\pi_{1}\left(\Delta-\mathcal{A}, \alpha_{0}\right)$, which maps $\eta^{\prime}$ to $\left(\eta^{\prime \prime} \eta^{\prime}\right) \eta^{\prime \prime}\left(\eta^{\prime \prime} \eta^{\prime}\right)^{-1}$ and $\eta^{\prime \prime}$ to $\left(\eta^{\prime \prime} \eta^{\prime} \eta^{\prime \prime}\right) \eta^{\prime}\left(\eta^{\prime \prime} \eta^{\prime} \eta^{\prime \prime}\right)^{-1}$. The fact that $\left(\sigma_{\gamma}^{3}, 1\right)$ belongs to $\Gamma(\phi)$ then follows directly from the observation that $\tau_{S^{\prime}} \tau_{S^{\prime \prime}}\left(S^{\prime}\right)$ is Hamiltonian isotopic to $S^{\prime \prime}$ and $\tau_{S^{\prime \prime}} \tau_{S^{\prime}}\left(S^{\prime \prime}\right)$ is Hamiltonian isotopic to $S^{\prime}$ (the geometric property underlying the braid relation $\left.\tau_{S^{\prime}} \tau_{S^{\prime \prime}} \tau_{S^{\prime}} \sim \tau_{S^{\prime \prime}} \tau_{S^{\prime}} \tau_{S^{\prime \prime}}\right)$.

The isotopy class $\rho\left(\sigma_{\gamma}^{3}, 1\right)$ admits a representative with support contained in the preimage of a small neighborhood $U_{\gamma}$ of $\gamma$, and because $\phi$ is trivial over $U_{\gamma}$ away from the vanishing cycles, we can again consider a universal local model for a neighborhood of the configuration of vanishing cycles. Consider 
the map $F=\left(F_{1}, F_{2}\right): \mathbb{C}^{n+1} \rightarrow \mathbb{C}^{2}$ defined by

$$
F\left(z_{1}, \ldots, z_{n+1}\right)=\left(z_{1}, z_{n+1}^{3}-3 z_{1} z_{n+1}+z_{2}^{2}+\cdots+z_{n}^{2}\right) .
$$

For $t=\epsilon \mathrm{e}^{i \theta}$, the restriction of $F$ to the hypersurface $X_{t}=F_{1}^{-1}(t)=\{t\} \times \mathbb{C}^{n}$ induces a Lefschetz fibration $F_{2}: X_{t} \rightarrow \mathbb{C}$, whose generic fiber $F_{2}^{-1}(u)$ is the smooth hypersurface $z_{n+1}^{3}-3 t z_{n+1}+z_{2}^{2}+\ldots+z_{n}^{2}=0$ in $\{t\} \times \mathbb{C}^{n}$. There are two singular fibers, corresponding to the critical values $\pm 2 t^{3 / 2}$. It is a classical fact that the two vanishing cycles intersect transversely in a single point $\left(F_{2}: X_{t} \rightarrow \mathbb{C}\right.$ is a Morsification of an $A_{2}$ singularity).

As $\theta$ varies from 0 to $2 \pi$, the two critical values of $F_{2}$ are exchanged by the braid $\sigma^{3}$, where $\sigma$ is the half-twist along the straight line segment joining the critical values. Therefore, by definition $\rho\left(\sigma^{3}, 1\right)$ is the monodromy of the trivial fibration $F_{1}: \mathbb{C}^{n+1} \rightarrow \mathbb{C}$ around the origin, hence $\rho\left(\sigma^{3}, 1\right)=1$. In other words, an $S^{1}$-family of symplectic Lefschetz pencils on $M$ realizing the cube of the half-twist along the arc $\gamma$ has trivial monodromy because it bounds a $D^{2}$-family of $\mathbb{C P}^{1}$-valued maps on $M$, one of which presents a degenerate critical point of type $A_{2}$ (this corresponds to $t=0$ in the local model).

Our last construction of elements in $\operatorname{Ker}(\rho)$ is easier to describe in the special case of 4 -manifolds. Assume that $\operatorname{dim} M=4$, and let $\delta:[0,1] \rightarrow \Sigma_{0}$ be an embedded arc with endpoints in $N$. Let $\tau_{\delta} \in \mathcal{B}\left(\Sigma_{0}, N\right)$ be the halftwist exchanging the two base points $\delta(0)$ and $\delta(1)$ along the arc $\delta$. Finally, let $\gamma:[0,1] \rightarrow \Delta \subset \mathbb{C P}^{1}$ be an arc with endpoints in $\mathcal{A}$, with a supporting pair $\left(\eta^{\prime}, \eta^{\prime \prime}\right)$, and denote by $S^{\prime}, S^{\prime \prime}$ the corresponding vanishing cycles.

Proposition 10.6. Assume that the vanishing cycles $S^{\prime}, S^{\prime \prime} \subset \Sigma_{0}-N$ satisfy the following properties: $(i) S^{\prime}$ intersects $\delta$ in exactly one point; (ii) $S^{\prime \prime}$ is Hamiltonian isotopic to $\tau_{\delta}\left(S^{\prime}\right)$. Then $\left(\sigma_{\gamma}, \tau_{\delta}\right) \in \Gamma(\phi)$ and $\rho\left(\sigma_{\gamma}, \tau_{\delta}\right)=1$.

Note that, since $\tau_{\delta}$ represents the trivial element in the mapping class group of $\Sigma_{0}$, the two vanishing cycles $S^{\prime}, S^{\prime \prime}$ are actually Hamiltonian isotopic in $\Sigma_{0}$ (but not in $\Sigma_{0}-N$ ). In fact, $\gamma$ is not a matching path for $\phi$, but it is a matching path for the blown up Lefschetz fibration, and the homology class of the associated Lagrangian sphere in the blowup $\hat{M}$ is the difference between the exceptional classes of the blowups at the base points $\delta(0)$ and $\delta(1)$.

Proof. The action of $\sigma_{\gamma}$ on $\pi_{1}\left(\Delta-\mathcal{A}, \alpha_{0}\right)$ maps $\eta^{\prime}$ to $\eta^{\prime \prime}$, and $\eta^{\prime \prime}$ to $\eta^{\prime \prime} \eta^{\prime}\left(\eta^{\prime \prime}\right)^{-1}$. To prove that $\left(\sigma_{\gamma}, \tau_{\delta}\right) \in \Gamma(\phi)$, it is sufficient to check that $L_{\theta}\left(\sigma_{\gamma^{*}}\left(\eta^{\prime}\right)\right)=$ $\left[S^{\prime \prime}\right]=\tau_{\delta}\left(L_{\theta}\left(\eta^{\prime}\right)\right)$, which follows from assumption (ii), and $L_{\theta}\left(\sigma_{\gamma *}\left(\eta^{\prime \prime}\right)\right)=$ $\tau_{S^{\prime \prime}}^{-1}\left(\left[S^{\prime}\right]\right)=\tau_{\delta}\left(L_{\theta}\left(\eta^{\prime \prime}\right)\right)$. In other terms, we have to check that $\tau_{S^{\prime \prime}}^{-1}\left(S^{\prime}\right)$ and $\tau_{\delta}\left(S^{\prime \prime}\right)$ are mutually Hamiltonian isotopic. This is easily accomplished, either by drawing a picture or by observing that $\tau_{\delta}^{2}$ is a Dehn twist and using the 
so-called lantern relation in the mapping class group of a sphere with four punctures to show that $\tau_{S^{\prime \prime}}\left(\tau_{\delta}^{2}\left(S^{\prime}\right)\right)$ is isotopic to $S^{\prime}$.

A local model for the automorphism $\left(\sigma_{\gamma}, \tau_{\delta}\right)$ is given by a family of pencils $F_{t}\left(t=\epsilon \mathrm{e}^{i \theta}\right)$ defined over a neighborhood of the origin in $\mathbb{C}^{2}$ by $F_{t}(x, y)=$ $\left(x^{2}+y^{2}-t\right) / x$. The pencil $F_{t}$ has two base points $\left(0, \pm t^{1 / 2}\right)$, and two critical points $\left( \pm i t^{1 / 2}, 0\right)$, associated to critical values $\pm 2 i t^{1 / 2}$. The smooth fibers are conics in $\mathbb{C}^{2}$, while the singular fibers $\left(x \mp i t^{1 / 2}\right)^{2}+y^{2}=0$ are unions of two lines, each containing one of the two base points; a closer examination shows that the roles of the two base points are exchanged when passing from one singular fiber to the other, so that the vanishing cycles differ by a half-twist in the fiber, as required.

When $\theta$ varies from 0 to $2 \pi$, the critical values of $F_{\epsilon \exp (i \theta)}$ are exchanged by a half-twist; moreover, if we consider a reference fiber $F_{t}^{-1}(\lambda)=\left\{x^{2}+\right.$ $\left.y^{2}-t=\lambda x\right\}$ for $|\lambda|>2 \epsilon^{1 / 2}$, the monodromy as $\theta$ varies from 0 to $2 \pi$ is trivial if one forgets the base points, but exchanges the two base points $\left(0, \pm t^{1 / 2}\right)$ by a half-twist in the fiber. Therefore, the family of pencils $\left(F_{\epsilon \exp (i \theta)}\right)$ is indeed a local model for the situation at hand. Since the monodromy of this family is trivial, we conclude that $\rho\left(\sigma_{\gamma}, \tau_{\delta}\right)=1$. In other words, an $S^{1}$-family of symplectic Lefschetz pencils on $M$ realizing the automorphism $\left(\sigma_{\gamma}, \tau_{\delta}\right)$ has trivial monodromy because it bounds a $D^{2}$-family of $\mathbb{C P}^{1}$-valued maps, one of which possesses a degenerate base locus (for $t=0$, the fibers of $F_{t}$ intersect at the origin with multiplicity 2).

Proposition 10.6 has a natural generalization in higher dimensions. Recall that, inside $\Sigma_{0}$, the base locus $N$ represents a class Poincaré dual to a multiple of the symplectic class. By varying $N$ inside a pencil, we can obtain families of pencils on $M$ in which the base locus changes by a Dehn twist (a transposition exchanging two points in the case $\operatorname{dim} N=0$ considered above). More precisely, assume $\Sigma_{0}$ contains a Darboux ball $B \subset \mathbb{C}^{n-1}$ inside which $N$ is the hypersurface $z_{1}^{2}+\cdots+z_{n-1}^{2}=\epsilon$, with $0<\epsilon \ll 1$. Choose a constant $\alpha$ such that $\epsilon \ll \alpha \ll 1$ and a smooth cut-off function $\varphi$ : $[0, \infty) \rightarrow$ $[0, \pi]$ with support in $[0,2 \alpha]$ and equal to $\pi$ over the interval $[0, \alpha]$. Then $\left(z_{1}, \ldots, z_{n-1}\right) \mapsto\left(\mathrm{e}^{i \varphi(|z|)} z_{1}, \ldots, \mathrm{e}^{i \varphi(|z|)} z_{n-1}\right)$ is a symplectomorphism of $\Sigma_{0}$, and admits a $C^{1}$-small perturbation which maps $N$ to itself via a Dehn twist along the $(n-2)$-sphere $N \cap \mathbb{R}^{n-1}$, thus defining an isotopy class in $\mathcal{B}\left(\Sigma_{0}, N\right)$. This construction can be thought of as a "symplectic half-twist" of $\Sigma_{0}$ along a Lagrangian disc with boundary in $N$. As in the 4-dimensional case, there are natural situations where the kernel of $\rho: \Gamma(\phi) \rightarrow \pi_{0} \operatorname{Symp}(M, \omega)$ contains elements of the form $(\sigma, \tau)$, where $\sigma \in B_{r}$ is a half-twist and $\tau$ is of the form we just described (see the next section).

10.3. Matching paths and projective duality. One of the main motivations for understanding "trivial" pencil automorphisms (the kernel of $\rho$ ), 
besides clarifying the relationship between pencil automorphisms and isotopy classes of symplectomorphisms, is to optimize the search for matching paths in a symplectic Lefschetz pencil. Indeed, we have the following obvious statement:

Proposition 10.7. If $\gamma:[0,1] \rightarrow \Delta$ is a matching path for the pencil $\phi$, associated to a Lagrangian sphere $S_{\gamma} \subset M$, and if $(b, g) \in \Gamma(\phi)$, then $b_{*}(\gamma)$ is also a matching path, and the corresponding Lagrangian sphere $S_{b_{*}(\gamma)}$ is isotopic to $\rho(b, g)\left(S_{\gamma}\right)$.

In particular, the action of the braids $b \in B_{r}$ for which there exists $g \in$ $\mathcal{B}\left(\Sigma_{0}, N\right)$ such that $(b, g) \in \operatorname{Ker}(\rho)$ defines an equivalence relation on the set of embedded arcs in $\Delta$ with endpoints in $\mathcal{A}$. Any arc equivalent to a given matching path is also a matching path, and the corresponding Lagrangian spheres are mutually isotopic.

From now on, we assume for simplicity that $M$ is Kähler. A triple of generic holomorphic sections of a sufficiently ample line bundle $L^{\otimes k}, k \gg 0$, determines a $\mathbb{C P}^{2}$-valued map $f$ with generic local models, defined outside of a complex codimension 3 base locus $Z \subset M$. (When $M$ is a complex surface, $Z$ is empty and $f: M \rightarrow \mathbb{C P}^{2}$ is a branched covering). The discriminant curve $D=\operatorname{crit}(f) \subset \mathbb{C P}^{2}$ is a complex plane curve with cusp and node singularities. For a generic point $p \in \mathbb{C P}^{2}$, the pencil of lines through $p$ determines a Lefschetz pencil structure on $M$ (by composition with $f$ ); the fibers are the preimages by $f$ of the lines in the pencil, and the singular fibers correspond to those lines through $p$ that are tangent to the curve $D$.

Introduce the dual projective plane $\left(\mathbb{C P}^{2}\right)^{*}$, which is the set of all projective lines in $\mathbb{C P}^{2}$, and let $D^{*} \subset\left(\mathbb{C P}^{2}\right)^{*}$ be the dual curve of $D$, consisting of all the lines tangent to $D$ in $\mathbb{C P}^{2}$. Generically the only singularities of $D^{*}$ are again nodes (corresponding to lines that are tangent to $D$ in two points) and cusps (corresponding to inflection points of $D$ ). A point $p^{*}$ in $\left(\mathbb{C P}^{2}\right)^{*}$ defines a hyperplane section $\Sigma_{p^{*}}=\overline{f^{-1}\left(L_{p^{*}}\right)} \subset M$, where $L_{p^{*}} \subset \mathbb{C P}^{2}$ is the line dual to $p^{*}$. This hyperplane section is smooth if and only if the point $p^{*}$ lies outside of $D^{*}$. A line $\ell^{*} \subset\left(\mathbb{C P}^{2}\right)^{*}$ defines a pencil of hyperplane sections (the preimages by $f$ of the pencil of lines through the point dual to $\ell^{*}$ in $\mathbb{C P}^{2}$ ), whose singular fibers correspond to the points of $\ell^{*} \cap D^{*}$ (while the smooth fibers correspond to the other points of $\ell^{*}$ ). This is a Lefschetz pencil (in the sense of Definition 1.1) if and only if $\ell^{*}$ intersects $D^{*}$ transversely at smooth points of $D^{*}$; otherwise the map to $\mathbb{C P}^{1}$ corresponding to $\ell^{*}$ presents non-generic singularities.

If we consider a 1-parameter family of lines $\ell_{t}^{*} \subset\left(\mathbb{C P}^{2}\right)^{*}, t \in S^{1}$, such that each line $\ell_{t}^{*}$ is transverse to $D^{*}$, then we obtain a family of Lefschetz pencils $\phi_{t}, t \in S^{1}$, whose monodromy gives an element $(b, g) \in \Gamma\left(\phi_{0}\right)$ by considering the motion of the critical values of $\phi_{t}$ as $t$ varies along $S^{1}$. Since the family of maps $\phi_{t}$ can be thought of as a map from $M \times S^{1}$ to $\mathbb{C P}^{1}$ (defined outside 
of the base loci), it is clear that the induced symplectomorphism of $M$ is trivial, i.e., $(b, g) \in \operatorname{Ker}(\rho)$.

Since the critical values of $\phi_{t}$ correspond to the points of $\ell_{t}^{*} \cap D^{*}$, the braid $b \in B_{r}$ is simply the braid monodromy of the degree $r$ plane curve $D^{*}$ with respect to the family of lines $\ell_{t}^{*}$. More precisely, assume for simplicity that the lines $\ell_{t}^{*}$ all pass through a generic point $q_{0} \in\left(\mathbb{C P}^{2}\right)^{*}-D^{*}$, and consider a linear projection $\pi:\left(\mathbb{C P}^{2}\right)^{*}-\left\{q_{0}\right\} \rightarrow \mathbb{C P}^{1}$ with pole $q_{0}$. Let $\mathcal{A} \subset \mathbb{C P}^{1}$ be the set of critical values of $\pi_{\mid D^{*}}$, i.e., the set of those fibers of $\pi$ which pass through the singular points of $D^{*}$ or fail to be transverse to $D^{*}$. Restricting ourselves to an affine subset $\mathbb{C} \subset \mathbb{C P}^{1}$ over which the fibration $\pi$ is trivial, we can define the braid monodromy of $D^{*}, \psi_{D^{*}}: \pi_{1}(\mathbb{C}-\mathcal{A}) \rightarrow B_{r}$, in the following manner: given a loop $\gamma: S^{1} \rightarrow \mathbb{C}-\mathcal{A}$, for each $t \in S^{1}$ the intersection $D^{*} \cap \pi^{-1}(\gamma(t))$ is a configuration of $r$ points in $\pi^{-1}(\gamma(t)) \simeq \mathbb{C}$; the motion of these $r$ points as $t$ varies determines a braid $\psi_{D^{*}}(\gamma) \in B_{r}$ (see e.g., $[\mathbf{1 7}]$ or $[\mathbf{4}]$ for more details). Now, if we consider the pencils associated to a family of lines $\ell_{t}^{*}=\overline{\pi^{-1}(\gamma(t))} \subset\left(\mathbb{C P}^{2}\right)^{*}$ for some loop $\gamma: S^{1} \rightarrow \mathbb{C}-\mathcal{A}$, then by definition we have $b=\psi_{D^{*}}(\gamma)$.

As a corollary of Proposition 10.7 and the above remarks, the image of any matching path for $\phi_{0}$ under the action of any element of the monodromy group $\operatorname{Im}\left(\psi_{D^{*}}\right) \subset B_{r}$ is also a matching path for $\phi_{0}$, and the corresponding Lagrangian spheres are mutually isotopic. Recall that the inclusion $i$ : $\ell_{0}^{*}-$ $\left(\ell_{0}^{*} \cap D^{*}\right) \hookrightarrow\left(\mathbb{C P}^{2}\right)^{*}-D^{*}$ induces a surjective homomorphism on fundamental groups, and by the Zariski-van Kampen theorem, the kernel of $i_{*}$ is generated by relations of the form $g \simeq b_{*}(g)$ for all $g \in \pi_{1}\left(\ell_{0}^{*}-\left(\ell_{0}^{*} \cap D^{*}\right)\right)$ and all $b \in \operatorname{Im}\left(\psi_{D^{*}}\right) \subset B_{r}$. The corresponding statement for matching paths is the following: if two embedded arcs in $\ell_{0}^{*}$ with endpoints in $\ell_{0}^{*} \cap D^{*}$ are isotopic as arcs in $\left(\mathbb{C P}^{2}\right)^{*}$ with endpoints in $D^{*}$, and if one of them is a matching path for $\phi_{0}$, then the other one is also a matching path. (Another way to see this result is to consider a universal family of hyperplane sections over $\left(\mathbb{C P}^{2}\right)^{*}$, and observe that with respect to this universal fibration the notion of matching path makes sense for arcs in $\left(\mathbb{C P}^{2}\right)^{*}$ with endpoints in $\left.D^{*}\right)$. Hence the problem of classifying matching paths up to the equivalence relation introduced at the beginning of this section reduces to the space of isotopy classes of arcs in $\left(\mathbb{C P}^{2}\right)^{*}$ with endpoints in $D^{*}$.

In this context, the braid monodromy of $D^{*}$ does not yield any new types of elements in the kernel of $\rho: \Gamma\left(\phi_{0}\right) \rightarrow \pi_{0} \operatorname{Symp}(M, \omega)$, but rather provides a geometric way of obtaining kernel elements of the form described in Propositions 10.5 and 10.6. Namely, a node of $D^{*}$ corresponds to a line in $\mathbb{C P}^{2}$ that is tangent to $D$ in two points, i.e., a hyperplane section of $M$ with two ordinary double points, indicative of the presence of two mutually disjoint vanishing cycles in the pencil $\phi_{0}$; the braid monodromy of $D^{*}$ around (the image by $\pi$ of) a node is the square of a half-twist, and corresponds to the 
situation described in Proposition 10.5 (a). Similarly, a cusp of $D^{*}$ corresponds to a line in $\mathbb{C P}^{2}$ that is tangent to $D$ at an inflection point, i.e., a hyperplane section of $M$ with an $A_{2}$ (cusp) singularity, obtained from a smooth hyperplane section by collapsing two vanishing cycles that intersect transversely once; the braid monodromy of $D^{*}$ around a cusp is the cube of a half-twist, which corresponds to the situation described in Proposition 10.5 (b). Finally, a line in $\left(\mathbb{C P}^{2}\right)^{*}$ which is tangent to $D^{*}$ corresponds to a pencil of lines in $\mathbb{C P}^{2}$ passing through a point $p$ of $D$, i.e., to a pencil of hyperplane sections in $M$ whose base locus $f^{-1}(p)$ presents an ordinary double point (or, when $M$ is a complex surface, a base point with multiplicity 2 ); the braid monodromy around a tangency of $D^{*}$ with the fibers of $\pi$ is a half-twist, and corresponds to the situation discussed in Proposition 10.6.

The above discussion should extend to the case of arbitrary symplectic manifolds, using approximately holomorphic maps $f: M \rightarrow \mathbb{C P}^{2}$ determined by triples of sections of $L^{\otimes k}$ (cf. [4]) satisfying suitable additional transversality conditions. Although in this context the discriminant curve $D \subset \mathbb{C P}^{2}$ is no longer a complex curve, a "dual curve" $D^{*}$ may still be defined by considering suitable expressions involving the pseudoholomorphic part of the jet of the map $f$. It follows from a general result about estimated transversality for approximately holomorphic jets $[\boldsymbol{5}]$ that we can impose conditions on the map $f$ which ensure that $D^{*}$ is a well-defined symplectic curve in $\left(\mathbb{C P}^{2}\right)^{*}$ presenting complex cusps and nodes of either orientation as its only singularities. While the duality between $D$ and $D^{*}$ now only holds in a much weaker sense as in the complex case, it is still reasonable to expect that the braid monodromy of $D^{*}$ should give useful information about matching paths.

To finish the discussion, we mention the following

Conjecture 10.8. For pencils of sufficiently large degree $(k \gg 0)$, the kernel of the homomorphism $\rho: \Gamma\left(\phi_{k}\right) \rightarrow \pi_{0} \operatorname{Symp}(M, \omega)$ is generated by the three types of elements described in Propositions 10.5 and 10.6.

Motivation for this conjecture comes from the observation that an $S^{1}$ family of holomorphic pencils on a complex projective manifold $M \subset \mathbb{C P}^{N}$ can be described by the motion of a line inside the dual projective space $\left(\mathbb{C P}^{N}\right)^{*}$, and hence is related to the braid monodromy of the dual variety $M^{*} \subset\left(\mathbb{C P}^{N}\right)^{*}$; however, using Lefschetz hyperplane-type arguments, one can check that the braid monodromy of $M^{*}$ is generated by that of the intersection $D^{*}=M^{*} \cap\left(\mathbb{C P}^{2}\right)^{*}$ for a generic plane $\left(\mathbb{C P}^{2}\right)^{*} \subset\left(\mathbb{C P}^{N}\right)^{*}$. Also, in general, an $S^{1}$-family of Lefschetz pencils on $M$ whose monodromy belongs to the identity component in $\operatorname{Symp}(M, \omega)$ should extend to a $D^{2}$-family of $\mathbb{C P}^{1}$-valued maps, in which individual members may have singularities worse than those allowed in Lefschetz pencils: the phenomena that are expected to 
occur in complex codimension 1 are precisely those mentioned in the above discussion.

Finally, we would like to offer the speculation that a stronger form of Proposition 10.3 should hold for many symplectic manifolds, for instance if $\pi_{0} \operatorname{Symp}(M, \omega)$ is finitely generated: namely there may exist an integer $k_{0}$ such that $\rho$ is surjective for all $k \geq k_{0}$. In that case, by combining Proposition 10.3 with Conjecture 10.8 one would obtain a complete description of $\pi_{0} \operatorname{Symp}(M, \omega)$ in terms of pencil monodromy, and hence reduce in principle the problem of classifying isotopy classes of symplectomorphisms to a (prob-

ably inaccessible) combinatorial question, similarly to what can be expected from Theorem 1.3 for isotopy classes of Lagrangian spheres.

\section{References}

[1] J. Amoros, V. Muñoz and F. Presas, Generic behavior of asymptotically holomorphic pencils, J. Symplectic Geom. 2 (2004) 377-392

[2] D. Auroux, Asymptotically holomorphic families of symplectic submanifolds, Geom. Funct. Anal. 7 (1997), 971-995.

[3] D. Auroux, Symplectic 4-manifolds as branched coverings of $\mathbb{C P}^{2}$, Invent. Math. 139 (2000), 551-602.

[4] D. Auroux, Symplectic maps to projective spaces and symplectic invariants, Turkish J. Math. 25 (2001), 1-42.

[5] D. Auroux, Estimated transversality in symplectic geometry and projective maps, Symplectic Geometry and Mirror Symmetry (Seoul, 2000), 1-30, World Scientific, Singapore, 2001.

[6] D. Auroux, D. Gayet and J. P. Mohsen, Symplectic hypersurfaces in the complement of an isotropic submanifold, Math. Ann. 321 (2001), 739-754.

[7] D. Auroux and L. Katzarkov, A degree doubling formula for braid monodromies and Lefschetz pencils, preprint.

[8] J. Cerf, Sur les difféomorphismes de la sphère de dimension trois $\left(\Gamma_{4}=0\right)$, Lecture Notes in Math. 53, Springer, 1968.

[9] S. Donaldson, Symplectic submanifolds and almost-complex geometry, J. Differential Geom. 44 (1996), 666-705.

[10] S. Donaldson, Lefschetz pencils on symplectic manifolds, J. Differential Geom. 53 (1999), 205-236.

[11] R. Gompf, Toward a topological characterization of symplectic manifolds, J. Symplectic Geom. 2 (2004), 177-206.

[12] J. Milnor, Morse theory, Annals of Mathematics Studies, 51, Princeton University Press, Princeton, N.J., 1963.

[13] P. Seidel, Vanishing cycles and mutation, in Proceedings of 3rd European Congress of Mathematics (Barcelona, 2000), Vol. II, 65-85, Progr. Math. 202, Birkhäuser, Basel, 2001.

[14] P. Seidel, Lectures on four-dimensional Dehn twists, preprint math.SG/0309012.

[15] S. Smale, On the structure of manifolds, Amer. J. Math. 84 (1962), 387-399. 
[16] I. Smith, Lefschetz pencils and divisors in moduli space, Geom. Topol. 5 (2001), 579-608.

[17] M. Teicher, Braid groups, algebraic surfaces and fundamental groups of complements of branch curves, in 'Algebraic Geometry' (Santa Cruz, 1995), 127-150, Proc. Sympos. Pure Math., 62 (part 1), Amer. Math. Soc., Providence, 1997.

Department of Mathematics

M.I.T.

Cambridge, MA 02139-4307

E-mail address: auroux@math.mit.edu

Instituto de Matemáticas y Física Fundamental

Consejo Superior de Investigaciones Científicas

C/ Serrano 113bis

28006 MADRID, SPAIN

E-mail address: vicente.munoz@imaff.cfmac.csic.es

Departamento de Matemáticas

UNIVERSIDAD AUTÓNOMA DE MADRID

28049 MADRID, SPAIN

E-mail address: francisco.presas@uam.es

We are grateful to Paul Seidel for telling us a proof of Lemma 8.4. Many thanks to the referee for pointing us the possibility of the existence of exotic Lagrangian spheres. Also thanks to Luis Guijarro and Jesús Gonzalo for useful comments. The first author was partially supported by NSF grant DMS-0244844. The second author was partially supported by CICYT BFM2000-0024. The third author was partially supported by CICYT BFM2000-0024 and by a Post-doctoral Fellowship from Ministerio de Educación y Cultura of Spain. All authors were partially supported by The European Contract Human Potential Programme, Research Training Network HPRN-CT-2000-00101. 
